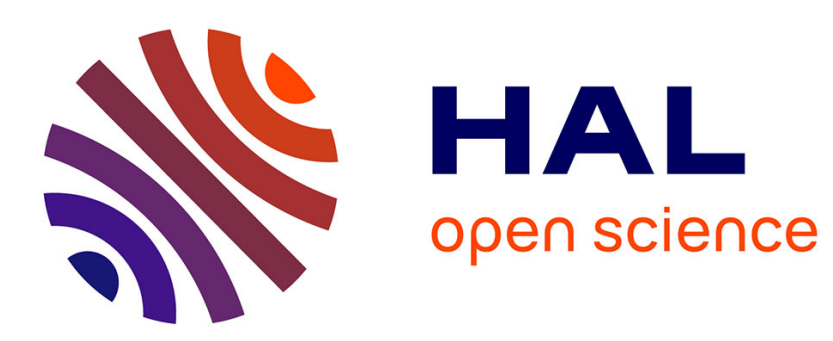

\title{
Determination of the transmissivity of a heterogeneous anisotropic fracture in slip flow conditions
}

\author{
Tony Zaouter, Didier Lasseux, Marc Prat
}

\section{To cite this version:}

Tony Zaouter, Didier Lasseux, Marc Prat. Determination of the transmissivity of a heterogeneous anisotropic fracture in slip flow conditions. Physical Review E , 2019, 100, pp.033115. 10.1103/PhysRevE.100.033115 . cea-02296537

\section{HAL Id: cea-02296537 https://hal-cea.archives-ouvertes.fr/cea-02296537}

Submitted on 25 Sep 2019

HAL is a multi-disciplinary open access archive for the deposit and dissemination of scientific research documents, whether they are published or not. The documents may come from teaching and research institutions in France or abroad, or from public or private research centers.
L'archive ouverte pluridisciplinaire HAL, est destinée au dépôt et à la diffusion de documents scientifiques de niveau recherche, publiés ou non, émanant des établissements d'enseignement et de recherche français ou étrangers, des laboratoires publics ou privés. 


\title{
Determination of the transmissivity of a heterogeneous anisotropic fracture in slip flow conditions
}

\author{
Tony Zaouter, ${ }^{1,2}$ Didier Lasseux $\odot,{ }^{3, *}$ and Marc Prat ${ }^{2}$ \\ ${ }^{1} C E A, D E N$, SEAD, Laboratoire d'Étanchéité, 30207 Bagnols-sur-Cèze, France \\ ${ }^{2}$ Institut de Mécanique des Fluides de Toulouse, IMFT, Université de Toulouse, 31400 Toulouse, France \\ ${ }^{3}$ CNRS, Institut de Mécanique et d'Ingénierie, I2M, UMR 5295-Esplanade des Arts et Métiers, 33405 Talence, Cedex, France
}

(Received 18 March 2019; published 23 September 2019)

\begin{abstract}
Rough fractures often exhibit a broad spectrum of defect length scales ranging from the microscopic (roughness) scale to a macroscopic one (waviness) and further to the megascopic scale corresponding to the entire fracture. The influence of these multiple scales and their reciprocal interactions are expected to play a significant role on the transport properties at the megascale. Focusing on the pressure-driven slightly compressible gas slip flow, a two-scale method is presented allowing the determination of the global transmissivity of a fracture on the basis of an upscaled Reynolds model. This model is applied on a tessellation of the fracture, each tile being affected by a macroscopic transmissivity tensor which encompasses the microscale transport information as a result of the first upscaling process. Then, the megascale flow problem in this structure, made of a set of tiles characterized by a heterogeneous and anisotropic transmissivity tensor field, is solved using a boundary element method. Numerical results obtained with this two-scale method are compared to the transmissivity computed with direct simulations carried out at the microscale on the whole fracture. This is performed on two model rough fractures, namely, a spiral groove and a fractal fracture, while varying their mean apertures to investigate a wide range of the average Knudsen number characteristic of the flow at the megascale. A good agreement is obtained between the two approaches showing the robustness of the two-scale method to determine the global transmissivity of the fracture while significantly reducing the overall computational time.
\end{abstract}

DOI: 10.1103/PhysRevE.100.033115

\section{INTRODUCTION}

Pressure-driven gas flow between confined rough walls (i.e., in a fracture) occurs in many industrial applications ranging from gas recovery through fractured rocks [1] to the leak rate determination of static mechanical seals [2-7]. In such applications, knowledge of the transport properties of the fractures represents a key issue as it can be decisive for the operating conditions of the whole process. A real rough fracture is generally characterized by a heterogeneous and multiscale aperture field and possible localized contact spots. Consequently, many different length scales are often to be considered as the dimensions of the fracture $L_{f}$ can be of the order of a few millimeters to few decimeters or more, while details at the roughness scale $l_{\beta}$ of micrometer or less in characteristic size, together with their correlation length may be of prominent impact on the flow [8-10]. Direct numerical modeling of the flow in this connected topography can therefore be a very challenging task and is often not desirable in practice, as it involves characteristic length scales that span over several orders of magnitude and sometimes an extremely large lateral extends to thickness aspect ratio.

In many practical situations, heterogeneity of the aperture field features very distinct and ordered characteristic length scales $L_{i}, i=1, \ldots, n$, that are well separated from each other, that is, $l_{\beta} \ll L_{i} \ll L_{f}$. Under such circumstances, the difficulty of solving the flow at the scale $L_{f}$ using a description

\footnotetext{
*didier.lasseux@u-bordeaux.fr
}

only at the scale $l_{\beta}$ can be circumvented by deriving average flow models at the successive intermediate scales, starting from the governing equations at the scale $l_{\beta}$ and making use of upscaling procedures up to the final megascale $L_{f}$. Such a strategy was adopted in the context of surface lubrication by splitting the problem into two scales, the flow being described by the so-called Reynolds equation at the scale $l_{\beta}$ as will be further detailed in this article, a model which is formally valid provided the aperture variation $\Delta h$ at the scale $l_{\beta}$ is such that $\Delta h / l_{\beta} \ll 1$. In the case of a one-dimensional longitudinal or transverse roughness pattern, Christensen [11] derived a flow model based on statistical averaging of the Reynolds equation. For a more general roughness pattern, Patir and Cheng [12] developed an average Reynolds equation which includes scalar "flow factors" as a signature of the effects of roughness on the flow at the upper scale. Using a stochastic approach, Tripp [13] extended Patir and Cheng's concept to obtain a tensorial form of the average flow model. More formal upscaling techniques have been further developed to obtain an averaged Reynolds equation involving a tensorial transmissivity coefficient which linearly relates the pressure gradient to the vectorial flow rate per unit width of the fracture. In particular, the volume (or surface) averaging technique has been employed to obtain an upscaled Reynolds equation together with the ancillary closure problem yielding the transmissivity tensor in the case of an incompressible liquid flow [14] or slightly compressible slip-corrected gas flow [15].

When the scale hierarchy fails at some characteristic length of the defects, such an upscaling approach may be inefficient 
between this specific scale and the upper one and special techniques (making use of a nonlocal approach, for instance) are usually required. This is the case, for instance, for a class of fractures in geological formations where the heterogeneity of the aperture at large scale can force the flow to concentrate in a few preferential paths of least resistance [16]. This may also be encountered in the typical case of a fracture arising from the assembly of two machined surfaces employed in sealing applications [3]. Topological defects at the different scales result from the manufacturing process itself. Indeed, machining induces length-scale cutoffs which can give rise to large-scale defects such as waviness, whose characteristic size may be distinctly separated from smaller ones like roughness and asperities but not from the fracture scale $L_{f}[17,18]$. In addition, a significant local (and hence global) anisotropy in the transmissivity may also appear. In a recent work, experimental results, obtained in the context of sealing incompressible fluid, were compared to a model taking only into account waviness (without roughness), for the sake of simplicity, with the idea that roughness may not have a significant impact on the overall transmissivity [19]. This recent work suggests the limitation of such an approach. In the work of Pérez-Ràfols et al. [20], the problem was addressed with a two-scale (namely a local and global scale) stochastic model. Once the local transmissivity tensors are computed, the global-scale problem is solved using a finite-volume-like method. However, anisotropy was not fully taken into account as the effect of the change of orientation of the principal axes of the local transmissivity tensors (i.e., the effect of their extradiagonal terms) was neglected.

In this work, the focus is laid on one-phase pressure-driven flow of a slightly compressible fluid in a single fracture using a two-scale description of the defects. More precisely, it is meant that the aperture field is characterized by the two scales $l_{\beta}$ and a single scale of heterogeneity (for instance, waviness) $L_{1}$, respectively referred to as the microscopic and macroscopic scales in the remainder of the article. It is assumed that the fracture aperture is slowly varying at the scale $l_{\beta}$ for a Reynolds approximation to be valid, reducing the problem to two-dimensional (2D) (i.e., a surface field of local scalar transmissivity) at the microscopic scale. On the basis of this approximation, it is further assumed that a representative elementary surface (RES), denoted by $\mathcal{S}$, can be exhibited so that the microscopic problem can be upscaled by surface averaging over $\mathcal{S}$ for a continuous description at the scale $L_{1}$. A priori, this requires the length-scale constraint $l_{\beta} \ll L_{1}$, which is the only one considered in this work. In fact, it must be emphasized that no additional assumption is made on the existence or not of other larger characteristic heterogeneity length scales, nor on their separation for the method proposed in this work to apply. In particular, nothing specific is supposed on the contrast between $L_{1}$ and $L_{f}$. At the scale $L_{1}$, the flow is hence governed by an upscaled Reynolds model as shown elsewhere [15]. The objective is to develop a method operating at the scale of the entire fracture $L_{f}$, referred to as the megascale in the remainder of the article, which can remain heterogeneous and anisotropic at this level, while embedding the microscopic information in transmissivity tensors that are determined at the macroscopic scale. A special attention is dedicated to the complete integration of macroscale anisotropy in the final estimate of the transmissivity tensor at the megascale. In addition, in the context of gas flow in a confined space, rarefaction effect can significantly impact the mass and momentum transfer (i.e., the transmissivity) since the fracture aperture may not be extremely large compared to the fluid mean free path at the pressure and temperature under consideration. This effect can be evaluated by the Knudsen number Kn, defined as the ratio of the mean free path of the fluid to a characteristic constriction length. When this dimensionless number is small enough compared to unity, typically in the range $10^{-2} \lesssim \mathrm{Kn} \lesssim 10^{-1}$, the flow takes place in the slip regime for which the continuum NavierStokes equations are still relevant provided a slip boundary condition is employed at the solid-fluid interface instead of the classical no-slip condition [21]. This situation has been of considerable interest for flow in microfluidics devices (see, for instance, [22,23]) or for the derivation of upscaled gas flow models in porous media (see [15,24-26]), with the purpose of extending the validity of the continuum no-slip flow models (typically for $\mathrm{Kn} \lesssim 10^{-2}$ ) to the slip regime. The method proposed here takes into account slip effects.

This paper is organized as follows. In Sec. II, the methodology is detailed. The different scales and governing equations for the process under study are reported and the numerical tool, relying on a boundary element method employed to solve the problem at the megascale, is presented. In Sec. III, examples of numerical results are provided starting with a comparison with analytical solutions on model configurations. Model rough fractures are further employed to assess the validity and robustness of the present method by a comparison with results from reference direct numerical simulations carried out at the microscopic scale. Conclusions of this study are provided in Sec. IV.

\section{TWO-SCALE METHOD}

The situation considered in this work is that of a stationary isothermal slightly compressible pressure-driven slip flow of a single barotropic fluid in a fracture. This fracture is assumed to result from two rough surfaces brought close to each other leaving a percolating aperture field, $h(x, y)$, defined as the distance between these two surfaces in a reference set of coordinates. Possible contact between these two surfaces can occur at specific locations, where $h(x, y)=0$. Heterogeneities of the aperture field are supposed to be organized so that the microscopic (roughness) scale is the smallest one having a characteristic length $l_{\beta}$, much smaller than that of all other possibly existing heterogeneity while $l_{\beta} \ll L_{f}$. A two-scale method is adopted considering the three relevant characteristic length scales reported in Fig. 1: The microscopic scale $l_{\beta}$, the macroscopic scale $L_{1}$, and the megascopic (fracture) scale $L_{f}$. The microscale and macroscale are supposed to be sufficiently well separated so that a continuous description can be made at the latter by integration of the microscale model over a RES containing all the necessary $l_{\beta}$-topological information, so that a local tensorial transmissivity can be identified. In practice, $L_{f}$ is the scale at which the megascopic boundary conditions are specified. 


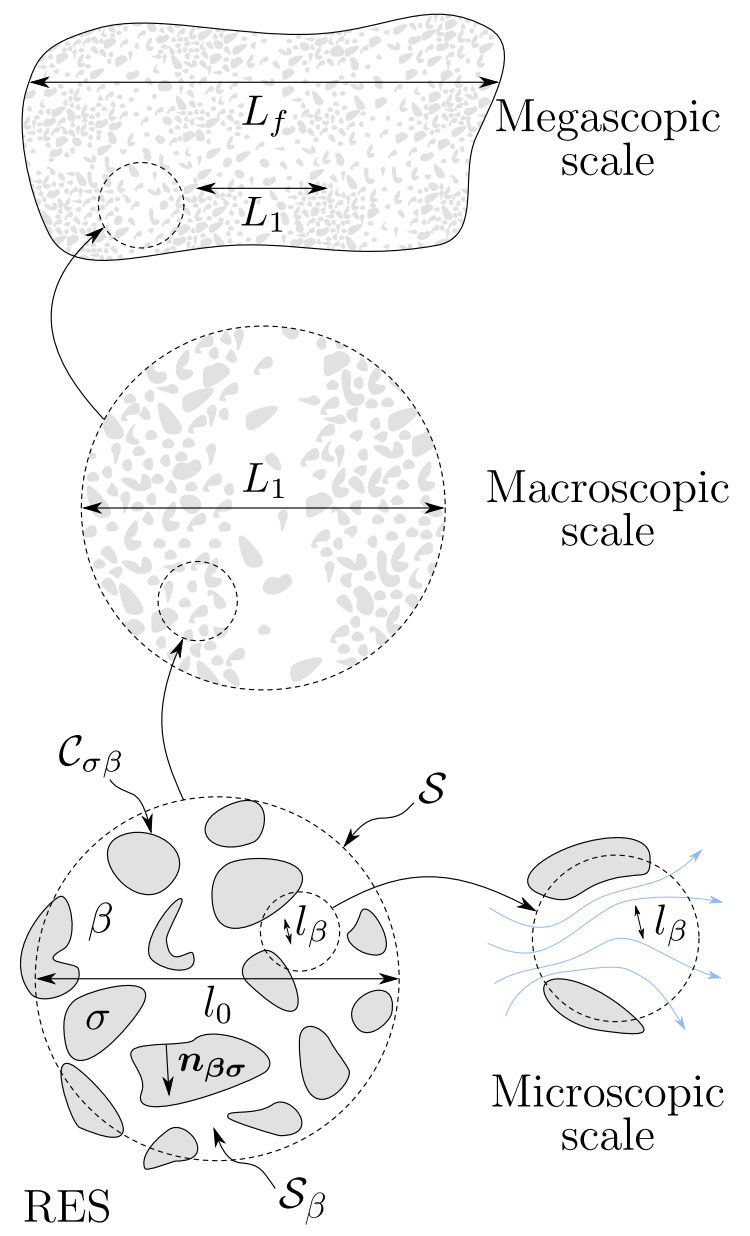

FIG. 1. Schematic view of the different scales of the fracture for the problem. $\beta$ is the fluid phase and the contact zones are denoted by $\sigma$. Note that the only length-scale constraint is $l_{\beta} \ll L_{1}$.

The objective is hence to compute the flow at the megascale and derive the transmissivity of the entire fracture using the field of transmissivity tensors at the macroscopic scale. The transmissivity tensors are obtained from the solution of the ancillary closure problem associated to the upscaling procedure applied to the Reynolds flow model operating at the microscale, and may contain the heterogeneity and local anisotropy inherent to the aperture field, together with the local slip effects.

\section{A. Governing equations}

In the remainder of this article, it is assumed that the fluid-structure interaction can be neglected and that no relative sliding motion between the surfaces occurs. Moreover, the aperture field is supposed to slowly vary with the in-plane coordinates. Under these circumstances, and when gravity effects can be disregarded with respect to the viscous forces, the Reynolds equation can be used to describe the flow at the microscopic scale [15,27]. Its validity may be questionable when the assumption of a smoothly varying aperture field does not hold, as discussed in [28]. This states that Reynolds equation remains valid up to a certain scale. This may be a particularly constraining situation for a fracture with a broad spectrum of frequencies (e.g., a self-affine fracture), where the local slopes diverges at a sufficiently small scale [8]. In the slip flow regime, the Reynolds model takes the form

$$
\begin{aligned}
\nabla \cdot \boldsymbol{q} & =0 \quad \text { in } \mathcal{A}_{\beta}, \\
\boldsymbol{q} & =-\rho \frac{h^{3}}{12 \mu}(1+6 \xi \mathrm{Kn}) \nabla p \quad \text { in } \mathcal{A}_{\beta}, \\
\rho & =\varphi(p) \quad \text { in } \mathcal{A}_{\beta}, \\
\boldsymbol{q} \cdot \boldsymbol{n}_{\boldsymbol{\beta} \boldsymbol{\sigma}} & =0 \quad \text { on } \mathcal{C}_{\sigma \beta} .
\end{aligned}
$$

The complete derivation of this model was reported in [15] and the reader is referred to this article for the details. In Eqs. (1), $\mathcal{A}_{\beta}$ designates the fluid-phase domain and $\mathcal{C}_{\sigma \beta}$ is the solid-fluid interface, that is, the contours of the contact zones that are referred to as $\sigma$. Moreover, $\boldsymbol{q}$ is the mass flow rate vector per unit width in each of the in-plane directions, $h$ is the local aperture of the fracture, $\rho$ is the fluid density, $p$ its pressure, and $\mu$ its dynamic viscosity, which is considered to be constant. The barotropic character of the fluid is reflected in the state equation (1c) whereas Eq. (1d) is a consequence of the impermeability of the contact zones, $\boldsymbol{n}_{\boldsymbol{\beta} \sigma}$ being the unit normal vector to the contours $\mathcal{C}_{\sigma \beta}$ directed from $\beta$ to $\sigma$ (see Fig. 1). The momentum equation (1b) was obtained using a first order slip boundary condition [29] involving the slip parameter $\xi$, that depends on the tangential momentum accommodation coefficient which is expected to be of the order of unity $[21,30]$. The momentum equation also includes the Knudsen number $\mathrm{Kn}=\lambda / h$, where $\lambda$ is the local mean free path of the gas molecules at the pressure and temperature under concern. If intermolecular collisions are assumed to be those between hard spheres, the mean free path can be expressed as [31]

$$
\lambda=\frac{M}{\pi \sqrt{2} \delta^{2} \mathcal{N}_{\mathrm{A}} \rho} .
$$

In this relationship, $M$ is the molar mass of the gas, $\mathcal{N}_{\mathrm{A}}$ is the Avogadro number, and $\delta$ denotes the effective collision diameter of the gas molecules. When the Knudsen number is everywhere vanishingly small, the usual no-slip Reynolds equation is recovered [32].

Focus is now laid upon the model that governs the flow at the macroscopic scale. Such a model can be derived by surface averaging the above microscopic boundary value problem, the method being completely similar to volume averaging [33]. This upscaling procedure, applied to Eqs. (1), was developed in a previous work by the authors and is briefly recalled in the following [15]. The averaging is carried out over a RES, $\mathcal{S}$, of surface $S$ and size $l_{0}$ (see Fig. 1) using the superficial and intrinsic averages, which, for any quantity $\psi$ defined in the $\beta$ phase, are respectively given by

$$
\langle\psi\rangle=\frac{1}{S} \int_{\mathcal{S}_{\beta}} \psi d S, \quad\langle\psi\rangle^{\beta}=\frac{1}{S_{\beta}} \int_{\mathcal{S}_{\beta}} \psi d S,
$$

$\mathcal{S}_{\beta}$ (of surface $S_{\beta}$ ) being the region occupied by the $\beta$ phase within $\mathcal{S}$. The RES is meant to contain all the necessary information of the defects at scale $l_{\beta}$ so that it can be characterized by its transmissivity which may, however, vary at the scale of defects $L_{1}$ immediately larger than $l_{\beta}$. In the context of sealing between clamped machined surfaces, $l_{\beta}$ can be identified as 
the characteristic length scale of roughness, as mentioned at the beginning of Sec. II, while $L_{1}$ is the typical length scale of waviness.

The upscaling process relies on a scale separation hypothesis, namely,

$$
l_{\beta} \ll l_{0} \ll L_{1} .
$$

Under the scale separation assumption, any quantity $\psi$ in $\mathcal{S}_{\beta}$ can be decomposed as the sum of an average value $\langle\psi\rangle^{\beta}$, which varies at the scale $L_{1}$ and a deviation $\widetilde{\psi}$, which varies at the scale $l_{\beta}$ [34]. To progress toward the macroscopic model, further hypotheses are employed. First, the slightly compressible flow assumption, expressed by $\widetilde{\rho} \ll\langle\rho\rangle^{\beta}$, is used so that $\rho \approx\langle\rho\rangle^{\beta}$ in the RES, corresponding to a locally incompressible flow, although it could still remain compressible at the scale $L_{1}$ (and $L_{f}$ ). Accordingly, a representative mean free path $\bar{\lambda}$ is introduced with the following expression, analogous to Eq. (2):

$$
\bar{\lambda}=\frac{M}{\pi \sqrt{2} \delta^{2} \mathcal{N}_{\mathrm{A}}\langle\rho\rangle^{\beta}} .
$$

Second, on the basis of the separation of length scales, the problem, expressed in terms of the pressure deviation, is made periodic, which means that the RES is considered as geometrically periodic and the gradient of the average pressure $\nabla\langle p\rangle^{\beta}$ is considered as constant on the RES. Under these circumstances, the macroscopic flow model, involving macroscopic quantities only, is given by [15]

$$
\begin{aligned}
\nabla \cdot\langle\boldsymbol{q}\rangle & =0, \\
\langle\boldsymbol{q}\rangle & =-\langle\rho\rangle^{\beta} \frac{\boldsymbol{K}}{\mu} \cdot \nabla\langle p\rangle^{\beta}, \\
\langle\rho\rangle^{\beta} & =\varphi\left(\langle p\rangle^{\beta}\right) .
\end{aligned}
$$

The momentum conservation equation (6b), which has a "Reynolds-type" form, linearly relates the average mass flow rate per unit width to the gradient of the average pressure, the proportionality coefficient being the transmissivity tensor $\boldsymbol{K}$ of the RES whose expression is

$$
\boldsymbol{K}=\langle k(\boldsymbol{I}+\nabla \boldsymbol{b})\rangle .
$$

Here, $\boldsymbol{I}$ is the identity tensor and $\boldsymbol{b}$ is a closure variable, solution of the following auxiliary (closure) microscale problem

$$
\begin{aligned}
\nabla \cdot(k\{\boldsymbol{I}+\nabla \boldsymbol{b}\}) & =0 \quad \text { in } \mathcal{S}_{\beta}, \\
\boldsymbol{n}_{\boldsymbol{\beta} \sigma} \cdot \nabla \boldsymbol{b} & =-\boldsymbol{n}_{\boldsymbol{\beta} \sigma} \quad \text { on } \mathcal{C}_{\sigma \beta}, \\
\boldsymbol{b}\left(\boldsymbol{x}+\boldsymbol{\Pi}_{\boldsymbol{i}}\right) & =\boldsymbol{b}(\boldsymbol{x}) \quad i=x, y .
\end{aligned}
$$

In this problem, $\boldsymbol{\Pi}_{\boldsymbol{i}}$ represents the two periodic lattice vectors of the unit cell (RES) and $k$ is the local transmissivity (which varies at the scale $l_{\beta}$ ) involved in Eq. (1b) and given by

$$
k=\frac{h^{3}+6 \xi \bar{\lambda} h^{2}}{12} .
$$

It must be noted that the flow problem is reduced to $2 \mathrm{D}$ on a surface characterized by the local transmissivity $\boldsymbol{K}$. For this reason, the term surface will be employed in the remainder of the article to refer to the fracture.
The transmissivity tensor $\boldsymbol{K}$ is obviously not intrinsic as it does not only depend on the microstructure (i.e., the $h$ field), but also on the representative mean free path $\bar{\lambda}$ on the RES, present in the expression of $k$. In the absence of slip effects, this tensor becomes intrinsic to the microstructure, a result extensively used in the study of liquid flows in fractures $[4,14,20]$.

Prediction of the flow at the megascale represents a new difficulty that is dealt with using the two-scale method detailed in Sec. II B below.

\section{B. General procedure of the method}

The field of $h$, the average density $\langle\rho\rangle^{\beta}$, as well as the representative mean free path $\bar{\lambda}$ are expected to vary at the scale $L_{1}$, which is likely to be less than or of the same order as $L_{f}$. Consequently, $\boldsymbol{K}$ will undoubtedly experience some variations over the whole fracture. The combination of all these important physical features makes the determination of the transmissivity of the entire heterogeneous fracture very difficult. In particular, the principal axes of the tensor $\boldsymbol{K}$ can be very different from one location to another within the fracture. In contrast with the finite element of finite volume methods, the boundary element method used here is a very elegant and efficient way of dealing with this situation. The procedure to simulate the flow at the megascale using the two-scale method is summarized in the flowchart of Fig. 2 where the main steps were schematized.

Without loss of generality, the fracture is supposed to be a rectangular domain of dimensions $L_{x}$ and $L_{y}$ in the $x$ and $y$ directions, respectively. For a contact having an annular shape, a situation of wide interest for many sealing applications $[3,6,19]$, this may be thought of resulting from unrolling the ring so that the problem can be treated in Cartesian coordinates, an approximation which remains valid provided the width of the contact is much smaller than its circumference (i.e., $\left.L_{x} / L_{y} \ll 1\right)$. The dimension $L_{y}$ defines the scale $L_{f}$. The main steps of the two-scale method can be described as follows.

(1) The aperture field of the entire fracture to be studied, $h(x, y)$, is the basic input of the procedure. For further numerical treatment, it is supposed to be known on a regular structured grid composed of $n_{x} \times n_{y}$ grid blocks, $n_{x}$ and $n_{y}$ being the grid size in the $x$ and $y$ directions, respectively. In practice, this aperture field may be obtained in various ways, for instance, numerically from contact mechanics computation between rough surfaces $[4,20]$.

(2) The next step is to subdivide the megascopic domain into a given number of rectangular subdomains, referred to as tiles in the following. This tessellation is supposed to be composed of $n_{p}=n_{p x} \times n_{p y}$ tiles, $n_{p x}$ and $n_{p y}$ being the number of tiles in the $x$ and $y$ directions, respectively (in the example of Fig. 2, $n_{p x}=3$ and $n_{p y}=2$ ). Each tile encompasses a set of the total number of points of the fracture and can be viewed as a local RES. The choice of $n_{p x}$ and $n_{p y}$ therefore remains a degree of freedom in the procedure and has to be guided by the underlying geometry of the aperture field, with the idea that the constraint expressed in (4) must be satisfied, $l_{0}$ being considered as the characteristic dimension of the tile. 


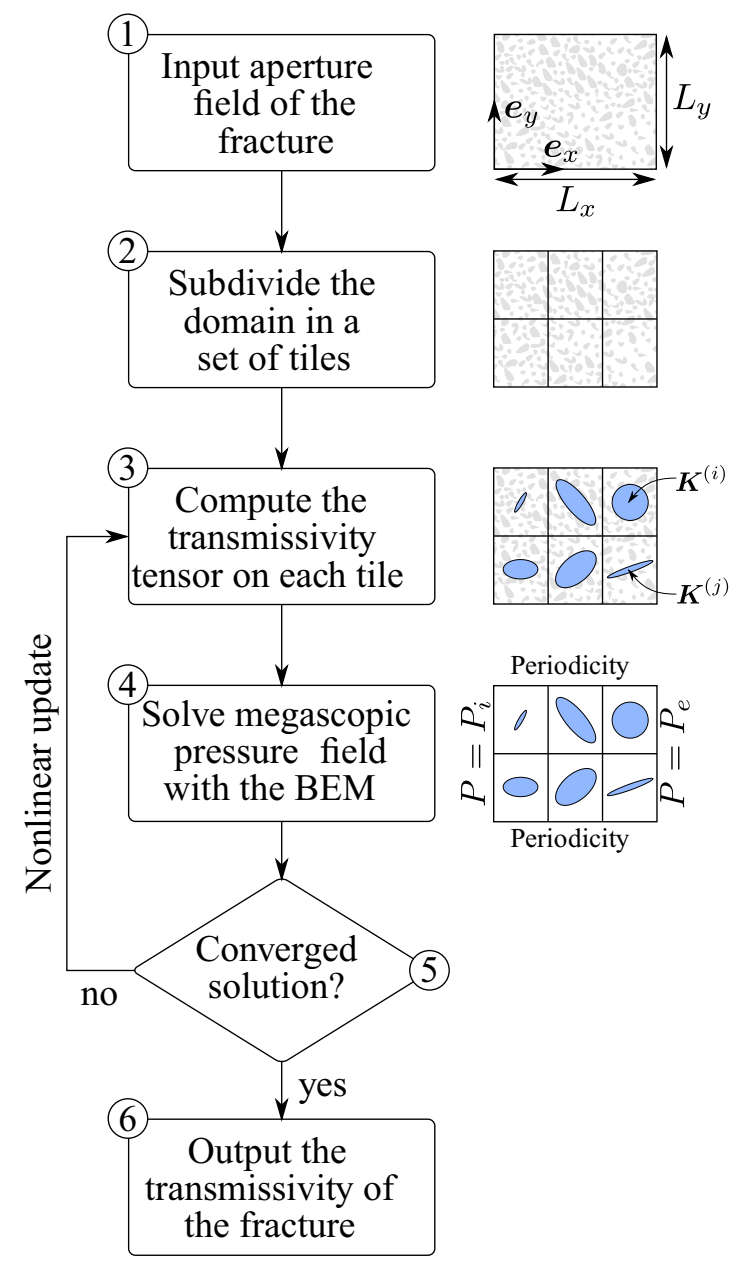

FIG. 2. Flowchart of the two-scale method. Anisotropy of the transmissivity tensors (steps 3 and 4 ) is schematized by ellipses which minor and major axes materialize tensors principal axes with different orientations.

(3) Each tile being viewed as a RES, the flow model is the macroscopic one given in Eqs. (6). In addition, the average density $\langle\rho\rangle^{\beta}$ and representative mean free path $\bar{\lambda}$ are taken as constants on each tile, although different from one tile to another. The transmissivity tensor on each tile is hence computed using Eq. (7) after the closure problem in Eqs. (8) is solved. This is performed using a finite volume discretization scheme which is second order in space [35], and the resulting linear system is solved with the conjugate gradient method. The algorithm is the one used by Zaouter et al. [15]. It can be used in a full parallel way since tensors on the tessellation are totally independent from each other. In Fig. 2, the transmissivity tensor on each tile was schematically represented by an ellipse whose minor and major axes depict its principal axes with their own orientation. At the end of this step, the tessellation of the megascale domain is characterized by a piecewise constant, heterogeneous, and anisotropic transmissivity tensor field. Flow at the megascale can hence be predicted from the solution of the Reynolds equation on this heterogeneous field of tensorial transmissivities.

(4) The flow problem in the resulting transmissivity tensorial field given by Eqs. (6) is solved ensuring the continuity of the average pressure $\langle p\rangle^{\beta}$ and normal flux $\langle\boldsymbol{q}\rangle \cdot \boldsymbol{n}$ at the boundaries between two adjacent tiles. The solution is sought on the pressure field using a boundary element method (BEM) presented in more details in Sec. II C. This method takes into account the heterogeneous and fully anisotropic character of the tensorial transmissivity field. Boundary conditions at the megascale correspond to a pressure drop applied in the $x$ direction resulting from Dirichlet boundary conditions (namely, an internal and external pressure $P_{i}$ and $P_{e}$ ) at $x=x_{0}$ and $x_{0}+L_{x}$, respectively, while periodicity is assumed in the $y$ direction (see Fig. 2).

(5) The problem to be solved is nonlinear in nature. Indeed, density, considered as constant on each tile, depends on the corresponding average pressure and varies from one tile to another, and so does $\bar{\lambda}$ on which transmissivity tensors depend. To estimate the value of $\bar{\lambda}$ on a tile, one first needs the mean pressure on this tile, denoted $\widehat{p}$, taken as the average of the internal pressure field computed with the BEM in step 4. The mean density can be estimated from a first order approximation $\widehat{\rho}=\varphi(\hat{p})$ of the state equation [25] and the representative mean free path can be deduced from Eq. (5). This is achieved by iterating on steps 3 and 4 from which the transmissivity tensor and mean pressure fields are, respectively, updated from iteration to iteration until convergence is reached on the field of $\widehat{p}$, starting from an initial guess and making use of a Picard iterative scheme [36]. In the absence of slip effects, the transmissivity tensor field is intrinsic and needs to be computed only once.

(6) Once convergence is reached, the total mass flow rate through the fracture can then be computed as indicated below (see last paragraph of Sec. IIC) and, from this result, the transmissivity $K$ of the entire fracture in the $x$ direction is deduced using the following one-dimensional (1D) megascale Reynolds equation

$$
\mathscr{Q}=\bar{\rho} \frac{K}{\mu} \frac{L_{y}}{L_{x}} \Delta P,
$$

where $\mathscr{Q}$ is the mass flow rate through the fracture, $\Delta P=$ $P_{i}-P_{e}$ is the imposed pressure drop, and $\bar{\rho}=\varphi(\bar{P})$ is the mean density, $\bar{P}=\left(P_{i}+P_{e}\right) / 2$ being the mean pressure. This last equation expresses the flux to force relationship resulting from the flow of an ideal gas in a homogeneous fracture of constant aperture having a transmissivity $K$ equivalent to the real one $[23,37]$.

To summarize, the procedure described above allows to split the problem in two scales that can be treated separately. Indeed, the microscopic information is first embedded into the local transmissivity tensor field that is employed to reconstruct the macroscopic scale information over the whole fracture. Using this macroscopic information, the heterogeneous resulting problem is then solved to obtain the transmissivity coefficient at the megascale.

Because effective contact between the two irregular surfaces may occur from place to place within the fracture, nonpercolating tiles having a zero transmissivity may appear. Such an occurrence is detected using a Hoshen-Kopelman's cluster labeling algorithm [38] in order to tag the corresponding tiles. The same algorithm is further used prior to the BEM solution at the megascale in order to only preserve the 
clusters of percolating tiles, i.e., clusters which connect the two faces $x=x_{0}$ and $x_{0}+L_{x}$ considering Dirichlet and periodic boundary conditions in the $x$ and $y$ direction, respectively.

\section{BEM solution of the megascopic problem}

To complete the description of the two-scale procedure, more details shall be given on the boundary element method (BEM) used to solve the fully anisotropic flow problem at the megascale (step 4 above and in Fig. 2). A similar approach was followed for one-phase tridimensional groundwater flow in a multidomain porous medium with piecewise uniform transport properties [39-41].

Combination of the mass conservation and the Reynolds equations (6a) and (6b) operating on a given tile $\mathcal{T}$ yields the following governing equation for the intrinsic average pressure:

$$
\nabla \cdot Q=\nabla \cdot(-\Gamma \cdot \nabla P)=0,
$$

where, for simplicity of notations, $P$ and $Q=-\Gamma \cdot \nabla P$ are used to denote $\langle p\rangle^{\beta}$ and $\langle\boldsymbol{q}\rangle$, respectively, while $\boldsymbol{\Gamma}=\widehat{\rho} \boldsymbol{K} / \mu$ represents the conductance, a second order symmetric [42] and positive definite [43] tensor for which the following notation is employed hereafter:

$$
\boldsymbol{\Gamma}=\left[\begin{array}{ll}
\Gamma_{x x} & \Gamma_{x y} \\
\Gamma_{x y} & \Gamma_{y y}
\end{array}\right] .
$$

Since $\boldsymbol{\Gamma}$ is assumed to be constant on $\mathcal{T}$, Eq. (11) can be transformed into

$$
\begin{aligned}
\Gamma: \nabla \nabla P & =\Gamma_{x x} \frac{\partial^{2} P}{\partial x^{2}}+2 \Gamma_{x y} \frac{\partial^{2} P}{\partial x \partial y}+\Gamma_{y y} \frac{\partial^{2} P}{\partial y^{2}} \\
& =0 .
\end{aligned}
$$

Without any loss of generality, the methodology to treat the problem on a multidomain with piecewise uniform conductance tensors may be illustrated considering two adjacent tiles $\mathcal{T}^{(i)}$ and $\mathcal{T}^{(j)}$ sharing a boundary $\mathcal{A}_{i j}$ (see Fig. 3). The problem can be stated as follows:

$$
\begin{aligned}
\boldsymbol{\Gamma}^{(i)}: \nabla \nabla P_{i} & =0 \quad \text { in } \mathcal{T}^{(i)}, \\
P_{i} & =P_{j} \quad \text { at } \mathcal{A}_{i j}, \\
\boldsymbol{n}^{(i)} \cdot\left(\boldsymbol{\Gamma}^{(i)} \cdot \nabla P_{i}\right) & =-\boldsymbol{n}^{(j)} \cdot\left(\boldsymbol{\Gamma}^{(j)} \cdot \nabla P_{j}\right) \quad \text { at } \mathcal{A}_{i j}, \\
\boldsymbol{\Gamma}^{(j)}: \nabla \nabla P_{j} & =0 \quad \text { in } \mathcal{T}^{(j)},
\end{aligned}
$$

where $\boldsymbol{n}^{(k)}$ is the outwardly directed unit normal vector to $\mathcal{T}^{(k)}$. Equations (14b) and (14c) express the pressure and normal flux continuity between the two domains.

To progress toward a solution easily tractable with the BEM, two successive transformations are applied, namely, a rotation followed by a dilatation as illustrated in Fig. 3, allowing to convert Eq. (13) into a Laplace equation. For the sake of simplicity in presentation, the application of these two transformations is illustrated below for a single tile, keeping in mind, however, that they have to be applied separately for each tile.

The first transformation, a rotation of angle $\theta$, is used to express the conductance tensor $\boldsymbol{\Gamma}_{\star}$ in a new rotated basis

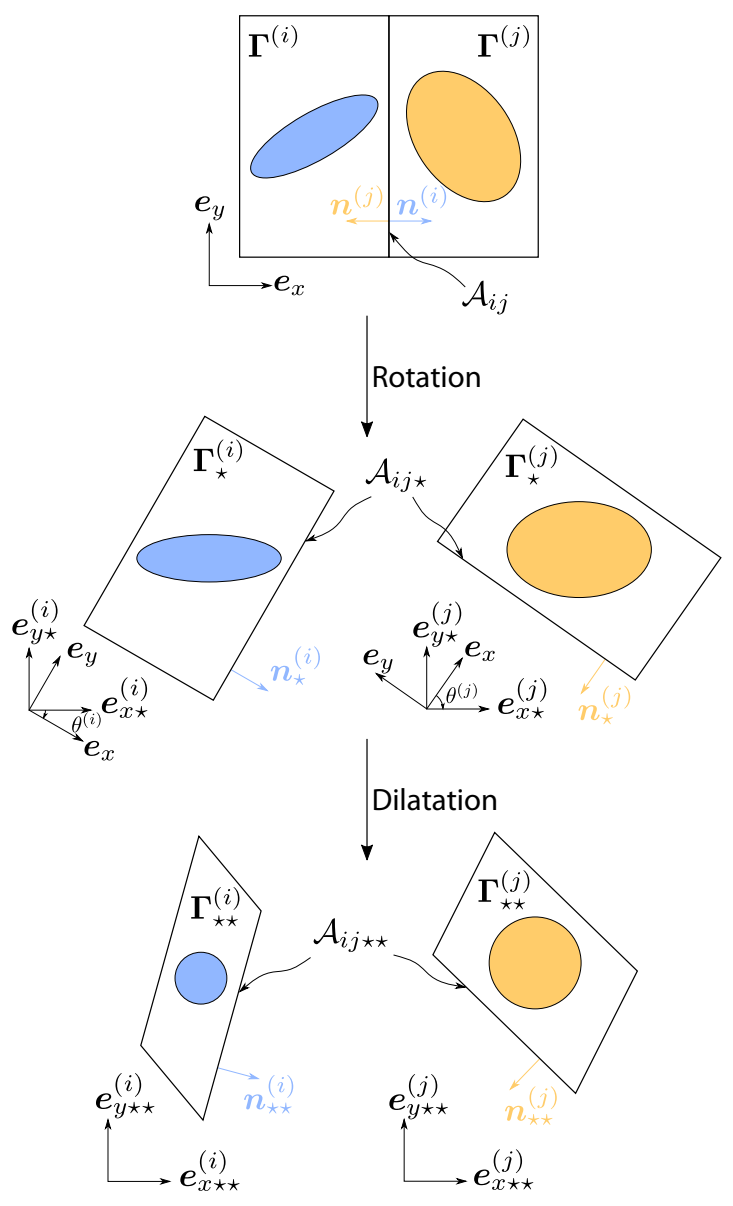

FIG. 3. Illustration of the two successive transformations (rotation and dilatation) applied to two adjacent tiles for the BEM flow solution in an anisotropic multidomain.

$\left(\boldsymbol{e}_{x \star}, \boldsymbol{e}_{y \star}\right)$ denoted with a star subscript symbol. A point, which position vector is $\boldsymbol{x}=x \boldsymbol{e}_{x}+y \boldsymbol{e}_{y}$ in the initial basis, will be positioned by $\boldsymbol{x}_{\star}=x_{\star} \boldsymbol{e}_{x \star}+y_{\star} \boldsymbol{e}_{y \star}$ in the new rotated basis. This change of basis can be formally written as

$$
\boldsymbol{x}_{\star}=\mathfrak{R}^{-1} \cdot \boldsymbol{x},
$$

where $\mathfrak{R}$ is the transformation matrix of a rotation of angle $\theta$ given by

$$
\mathfrak{R}=\left[\begin{array}{rr}
\cos \theta & -\sin \theta \\
\sin \theta & \cos \theta
\end{array}\right]
$$

When $\theta$ is appropriately chosen so that the principal axes of $\boldsymbol{\Gamma}$ coincide with $\left(\boldsymbol{e}_{x \star}, \boldsymbol{e}_{y \star}\right)$ and when Eq. (15) is employed, Eq. (13) is transformed into the following form:

$$
\Gamma_{1} \frac{\partial^{2} P}{\partial x_{\star}^{2}}+\Gamma_{2} \frac{\partial^{2} P}{\partial y_{\star}^{2}}=0
$$

with $\Gamma_{1}$ and $\Gamma_{2}$ the principal conductance values such that $\Gamma_{\star}$ may be written as

$$
\Gamma_{\star}=\left[\begin{array}{cc}
\Gamma_{1} & 0 \\
0 & \Gamma_{2}
\end{array}\right]
$$


in the basis $\left(\boldsymbol{e}_{x \star}, \boldsymbol{e}_{y \star}\right), \Gamma_{1}$ and $\Gamma_{1}$ being given by

$$
\begin{aligned}
\Gamma_{1} & =\frac{\Gamma_{x x}+\Gamma_{y y}}{2}+\sqrt{\left(\frac{\Gamma_{x x}-\Gamma_{y y}}{2}\right)^{2}+\Gamma_{x y}^{2},} \\
\Gamma_{2} & =\frac{\Gamma_{x x}+\Gamma_{y y}}{2}-\sqrt{\left(\frac{\Gamma_{x x}-\Gamma_{y y}}{2}\right)^{2}+\Gamma_{x y}^{2},} \\
\theta & =\frac{1}{2} \arctan \left(\frac{2 \Gamma_{x y}}{\Gamma_{x x}-\Gamma_{y y}}\right) .
\end{aligned}
$$

The second transformation is a dilatation of coefficient $\alpha$ along the $x_{\star}$ coordinate yielding an isotropic conductance tensor $\boldsymbol{\Gamma}_{\star \star}$ in the dilated basis $\left(\boldsymbol{e}_{x \star \star}, \boldsymbol{e}_{y_{\star \star}}\right)$ denoted with two star subscript symbols. The position vector $\boldsymbol{x}_{\star}$ in the rotated basis has coordinates $\boldsymbol{x}_{\star \star}=x_{\star \star} \boldsymbol{e}_{x \star \star}+y_{\star \star} \boldsymbol{e}_{y \star \star}$ in the new dilated basis and this can be expressed by the formal relationship

$$
\boldsymbol{x}_{\star \star}=\mathfrak{D}^{-1} \cdot \boldsymbol{x}_{\star},
$$

where $\mathfrak{D}$ is the transformation matrix of a $x_{\star}$ dilatation of coefficient $\alpha$ given by

$$
\mathfrak{D}=\left[\begin{array}{cc}
1 / \alpha & 0 \\
0 & 1
\end{array}\right]
$$

Provided the coefficient $\alpha$ is chosen such that

$$
\alpha=\sqrt{\frac{\Gamma_{2}}{\Gamma_{1}}}
$$

it can be shown that the use of Eq. (20) transforms Eq. (17) in the dilated basis, into a classical Laplace equation for a domain of isotropic conductance $\Gamma_{2}$, namely,

$$
\Gamma_{2}\left(\frac{\partial^{2} P}{\partial x_{\star \star}^{2}}+\frac{\partial^{2} P}{\partial y_{\star \star}{ }^{2}}\right)=\Gamma_{2} \nabla_{\star \star}^{2} P=0 .
$$

To solve the problem in the transformed distorted subdomains resulting from rotation and dilatation on each tile, boundary conditions in Eqs. (14b) and (14c) have to be rewritten accordingly. At corresponding points between the boundary in the physical domain (on $\mathcal{A}_{i j}$ ) and in the transformed domain (on $\mathcal{A}_{i j \star \star}$ ), the pressure remains unmodified. However, this is not the case for the normal fluxes [39-41]. The rotation does not introduce any modification in the normal fluxes and, hence, it can be shown that

$$
Q_{n}=Q_{n \star},
$$

$Q_{n}$ and $Q_{n \star}$ being the normal projections of the fluxes $\boldsymbol{Q}$ and $\boldsymbol{Q}_{\star}$ in the $\left(\boldsymbol{e}_{x}, \boldsymbol{e}_{y}\right)$ and $\left(\boldsymbol{e}_{x \star}, \boldsymbol{e}_{y \star}\right)$ bases, respectively. Conversely, as will be seen below, the dilatation induces a transformation on the normal vectors at each boundary due to the distortion of the domains, yielding a modification of the fluxes expressions. Hence, a relationship between $Q_{n \star}$ and $Q_{n \star \star}$ [this latter being the normal flux resulting from dilatation in the $\left(\boldsymbol{e}_{x \star \star}, \boldsymbol{e}_{y \star \star}\right)$ basis] is required.

Using the dilatation transform in Eq. (20), the vector flux $\boldsymbol{Q}_{\star \star}$ in the $\left(\boldsymbol{e}_{x \star \star}, \boldsymbol{e}_{y \star \star}\right)$ basis is obtained from $\boldsymbol{Q}_{\star}$ in the $\left(\boldsymbol{e}_{x \star}, \boldsymbol{e}_{y \star}\right)$ basis as

$$
\begin{aligned}
& Q_{x \star \star}=\alpha Q_{x \star}, \\
& Q_{y \star \star}=Q_{y \star}
\end{aligned}
$$

with the intuitive notation for the vector flux coordinates in the corresponding bases. Notice that the tangential vector $t_{\star \star}$ at the boundary is obtained from $t_{\star}$ using the same relationship. If $\boldsymbol{n}_{\star}$ and $\boldsymbol{n}_{\star \star}$ denote the outwardly directed unit normal vectors at the boundary in the $\left(\boldsymbol{e}_{x \star}, \boldsymbol{e}_{y \star}\right)$ and $\left(\boldsymbol{e}_{x \star \star}, \boldsymbol{e}_{y \star \star}\right)$ bases, respectively, one can write

$$
\begin{aligned}
Q_{n \star} & =Q_{\star} \cdot \boldsymbol{n}_{\star} \\
& =Q_{x \star} n_{x \star}+Q_{y \star} n_{y \star}, \\
Q_{n \star \star} & =Q_{\star \star} \cdot \boldsymbol{n}_{\star \star} \\
& =Q_{x \star \star} n_{x \star \star}+Q_{y \star \star} n_{y \star \star} .
\end{aligned}
$$

At this stage, it is important to notice that, since the dilatation does not preserve orthogonality, the normal $\boldsymbol{n}_{\star \star}$, unlike $\boldsymbol{t}_{\star \star}$, does not derive from $\boldsymbol{n}_{\star}$ with the transformation in Eq. (20) but is given by

$$
\boldsymbol{n}_{\star \star}=\frac{1}{C} \mathfrak{D}^{\mathrm{T}} \cdot \boldsymbol{n}_{\star},
$$

that is,

$$
\begin{aligned}
& n_{x \star \star}=\frac{1}{\alpha C} n_{x \star}, \\
& n_{y \star \star}=\frac{1}{C} n_{y \star} .
\end{aligned}
$$

The coefficient $C$ was introduced for $\boldsymbol{n}_{\star \star}$ to be a unit vector and is therefore such that

$$
C=\left\{\left(\alpha n_{x \star \star}\right)^{2}+n_{y \star \star}^{2}\right\}^{-1 / 2},
$$

and this expression is analogous to that obtained in a threedimensional configuration [40].

Introducing the relationships (25) and (28) into Eq. (26a) and identifying the result with Eq. (26b) provides the following relationship:

$$
Q_{n \star}=C Q_{n \star \star} .
$$

Since the normal derivative of the pressure is the given quantity on the boundary, one can write in the dilated basis [39]

$$
Q_{n \star \star}=-\Gamma_{2} \frac{\partial P}{\partial \boldsymbol{n}_{\star \star}} .
$$

Introducing Eqs. (30) and (31) back into Eq. (24) yields the following expression for $Q_{n}$ :

$$
Q_{n}=-\Gamma_{2} C \frac{\partial P}{\partial \boldsymbol{n}_{\star \star}} .
$$

Returning to the multidomain problem in Eqs. (14), applying the rotation and dilatation transformations described above for each domain allows to rewrite this problem as

$$
\begin{aligned}
\nabla_{\star \star}^{2} P_{i} & =0 \quad \text { in } \mathcal{T}_{\star \star}^{(i)}, \\
P_{i} & =P_{j} \quad \text { at } \mathcal{A}_{i j \star \star}, \\
\Gamma_{2}^{(i)} C^{(i)} \frac{\partial P_{i}}{\partial \boldsymbol{n}_{\star \star}^{(i)}} & =-\Gamma_{2}^{(j)} C^{(j)} \frac{\partial P_{j}}{\partial \boldsymbol{n}_{\star \star}^{(j)}} \quad \text { at } \mathcal{A}_{i j \star \star}, \\
\nabla_{\star \star}^{2} P_{j} & =0 \quad \text { in } \mathcal{T}_{\star \star}^{(j)} .
\end{aligned}
$$

To complete the problem statement, the outer boundary conditions at the megascale must be taken into account, 
namely, Dirichlet and periodic conditions in the $x$ and $y$ directions, respectively, as mentioned in Sec. II B, step 4 of the procedure.

Expressed in the rotated and dilated bases, the governing equations for the flow obey a Laplace problem with boundary conditions that are well adapted for a solution to be sought with a BEM. This is carried out with a boundary integral formulation on each tile using Green-Riemann's theorem [44]. Here, the boundary integrals were discretized using constant boundary elements on which the unknowns $P$ and/or $\partial P / \partial \boldsymbol{n}_{\star \star}$ are constant, equal to their central values. Integrals over each element were computed from their analytical expressions $[36,44]$. This yields a linear system providing, in a first step, the solution for $\partial P / \partial \boldsymbol{n}_{\star \star}$ on all the boundaries (including the internal interfaces between tiles and the outer megascale boundaries) and for $P$ on the internal interfaces and at $y=0$ and $L_{y}$. The linear system is relatively sparse with local dense blocks; it is also nonsymmetric, a typical situation with the BEM $[36,44]$. Consequently, the sparse multifrontal direct LU solver MUMPS $[45,46]$ was employed to solve this linear system.

At this point of the resolution where $\partial P / \partial \boldsymbol{n}_{\star \star}$ is known everywhere on the outer boundaries and tile interfaces, the normal mass flow rate per unit width on each element of the initial domain, $Q_{n}$, can be computed by making use of Eq. (32). Integration of $Q_{n}$ along the boundary of the whole fracture yields the total mass flow rate $\mathscr{Q}$, which, by making use of Eq. (10), is further employed to determine the transmissivity $K$ of the entire fracture as described in step 6 of the procedure (Sec. II B).

In a second step, the internal field of $P$ can be computed from the values of $P$ and $\partial P / \partial \boldsymbol{n}_{\star \star}$ on all the boundaries and tile interfaces using again the discretized integral formulation. Note that this step does not require a linear system solution. Taking the directional derivative of the integral equation for $P$ allows computing the internal fluxes in the transformed domain [44]. Finally, the internal fluxes can be expressed in the initial physical domain by inverting the dilatation and rotation transformations.

\section{NUMERICAL EXAMPLES}

\section{A. Validation on test cases}

In this section, a numerical validation of the method is performed on test cases for which the transmissivity coefficient of the entire fracture can be calculated analytically. Such situations are sparse and an example is when the transmissivity tensor field results from a serial or parallel arrangement of regions which individual transmissivity tensors have their principal directions aligned with the direction of the arrangement, leading to one-dimensional flow. To this end, a synthetic surface, composed of 15 tiles in the $x$ direction, as represented in Fig. 4, is considered in the absence of slip effects. On each tile, which is a square of size $l_{0}=1 \mathrm{~mm}$, the aperture varies in only one direction $t$ (being either $x$ or $y$ ), following a cosine function given by

$$
h(t)=h_{0}\left[1-\Upsilon \cos \left(\frac{2 \pi t}{l_{0}}\right)\right] .
$$
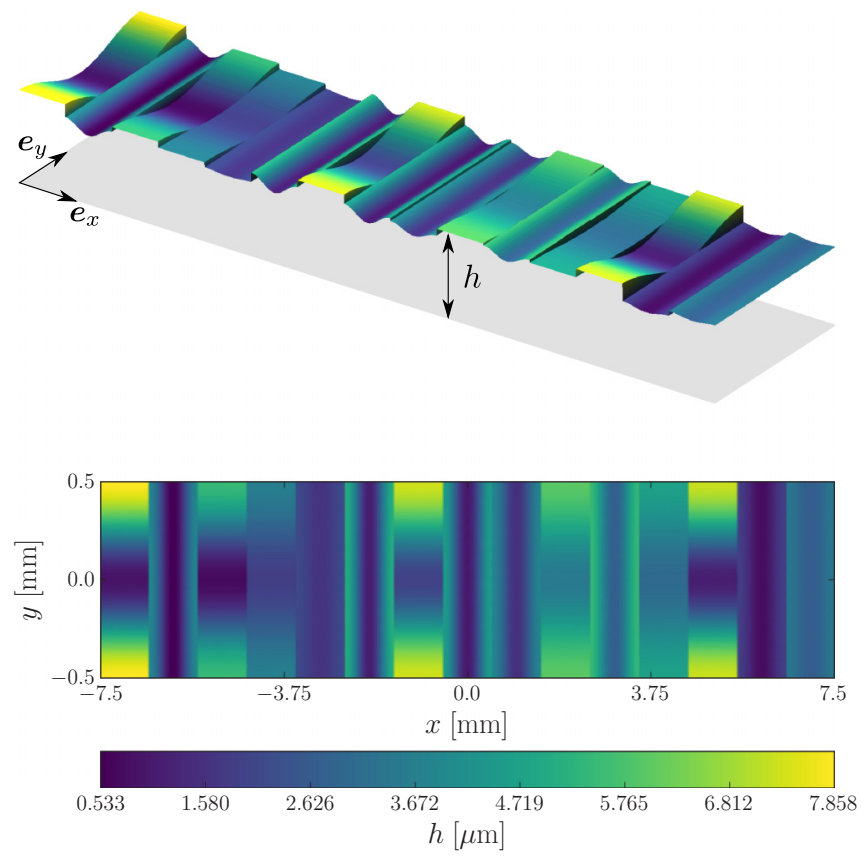

FIG. 4. Aperture field $h(x, y)$ of the synthetic surface composed of 15 "cosine tiles" of variable parameters. The serial configuration corresponds to the flow along $\boldsymbol{e}_{x}$. Top: 3D view. Bottom: $2 \mathrm{D}$ top view. Aspect ratio is distorted for clarity of presentation.

The mean aperture $h_{0}$, the amplitude $\Upsilon$, and the orientation $t$ are randomly chosen for each tile. Values of these parameters used in the following example are provided in Table I. The transmissivity tensor on each tile is diagonal and, under noslip condition, its components can be expressed as follows [15]:

$$
K_{\|}=\frac{2\left(1-\Upsilon^{2}\right)^{5 / 2}}{2+\Upsilon^{2}} \frac{h_{0}^{3}}{12}, \quad K_{\perp}=\frac{3 \Upsilon^{2}+2}{2} \frac{h_{0}^{3}}{12},
$$

TABLE I. Numerical values of the parameters used to generate the 15 "cosine tiles" of Fig. 4 (increasing tile number is from left to right in this figure). Transmissivity values are those in the absence of slip effects.

\begin{tabular}{llcccc}
\hline \hline Tile & $t$ & $\begin{array}{c}h_{0} \\
(\mu \mathrm{m})\end{array}$ & $\Upsilon$ & $\begin{array}{c}K_{x x} \\
\left(\mu \mathrm{m}^{3}\right)\end{array}$ & $\begin{array}{c}K_{y y} \\
\left(\mu \mathrm{m}^{3}\right)\end{array}$ \\
\hline 1 & $y$ & 4.385 & 0.792 & 13.64 & 0.454 \\
2 & $x$ & 2.253 & 0.763 & 0.083 & 1.786 \\
3 & $y$ & 3.098 & 0.764 & 4.646 & 0.215 \\
4 & $y$ & 2.774 & 0.318 & 2.049 & 1.296 \\
5 & $x$ & 1.918 & 0.147 & 0.551 & 0.607 \\
6 & $x$ & 3.138 & 0.636 & 0.584 & 4.138 \\
7 & $y$ & 4.656 & 0.574 & 12.57 & 2.653 \\
8 & $x$ & 2.829 & 0.637 & 0.426 & 3.036 \\
9 & $x$ & 2.723 & 0.429 & 0.925 & 2.147 \\
10 & $y$ & 4.757 & 0.258 & 9.863 & 7.305 \\
11 & $x$ & 4.114 & 0.332 & 4.108 & 6.758 \\
12 & $y$ & 3.864 & 0.214 & 5.136 & 4.182 \\
13 & $y$ & 4.211 & 0.727 & 11.15 & 0.753 \\
14 & $x$ & 1.371 & 0.430 & 0.118 & 0.274 \\
15 & $x$ & 3.073 & 0.127 & 2.302 & 2.476 \\
\hline \hline
\end{tabular}




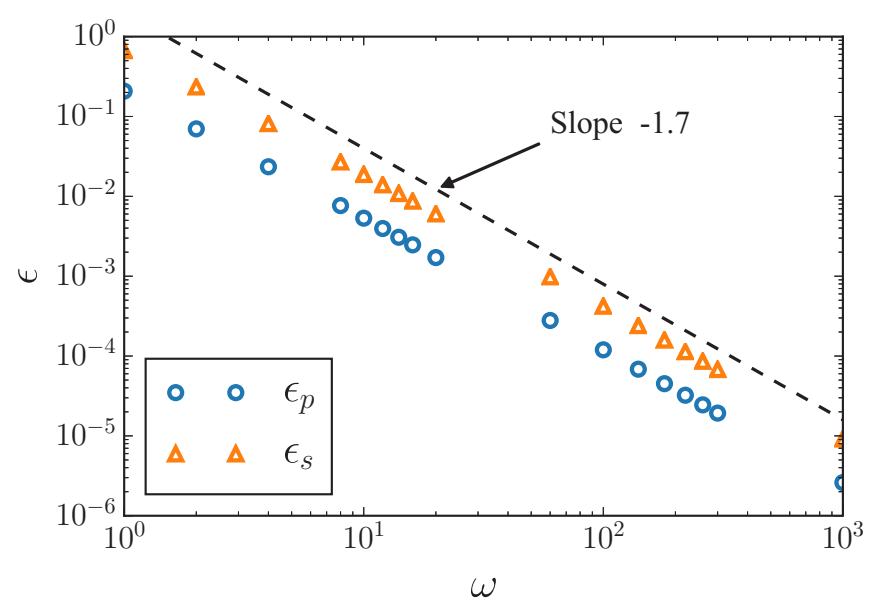

FIG. 5. Dependence of the relative error given in (37) between the analytical and computed solutions upon the mesh density $\omega$. The dashed line represents a power law function of $\omega$ with an exponent -1.7 .

where the symbols $\|$ and $\perp$ refer to the directions parallel and orthogonal to direction $t$, respectively. It can be noted that $K_{\perp} \geqslant K_{\|}$for values $0 \leqslant \Upsilon \leqslant 1$. Numerical values of these components are reported in Table I in the global reference frame of reference $\left(\boldsymbol{e}_{x}, \boldsymbol{e}_{y}\right)$ of Fig. 4 (that is for each tile, $K_{x x}=$ $K_{\|}$if $t \equiv x$ and $K_{x x}=K_{\perp}$ if $t \equiv y$, with a complementary nomenclature for $K_{y y}$ ). When a pressure gradient is applied along the $x$ or $y$ direction, the transmissivity of the fracture of Fig. 4 results from a set of transmissivities arranged in a serial or parallel configuration, respectively. Transmissivities of the fracture in serial configuration $K_{s}$ and in parallel configuration $K_{p}$ are given by the harmonic and arithmetic mean of the transmissivity field, respectively [37]. For the configuration under study, this reads as

$$
K_{s}=\left\langle K_{x x}^{-1}\right\rangle^{-1}, \quad K_{p}=\left\langle K_{y y}\right\rangle
$$

Using the numerical values of Table I, this gives $K_{s}=$ $0.510 \mu \mathrm{m}^{3}$ and $K_{p}=2.539 \mu \mathrm{m}^{3}$.

The objective is now to compare the transmissivities predicted by the two-scale method in the serial and parallel configurations, denoted $K_{s}^{\mathrm{BEM}}$ and $K_{p}^{\mathrm{BEM}}$, respectively, with the above analytical values. The two-scale method is employed considering an incompressible flow and Dirichlet boundary conditions such that $P_{i}=10^{5} \mathrm{~Pa}$ and $P_{e}=10^{4} \mathrm{~Pa}$. It must be noted that these conditions are applied at $x=x_{0}$ and $x_{0}+L_{x}$ (respectively $y=y_{0}$ and $y_{0}+L_{y}$ ) with periodicity in the $y$ (respectively $x$ ) direction to compute $K_{s}^{\mathrm{BEM}}$ (respectively $\left.K_{p}^{\mathrm{BEM}}\right)$. Here, $x_{0}=-7.5 \mathrm{~mm}, y_{0}=-0.5 \mathrm{~mm}, L_{x}=15 \mathrm{~mm}$, and $L_{y}=1 \mathrm{~mm}$. The relative error between the analytical and computed solutions for the serial configuration $\epsilon_{s}$ and the parallel configuration $\epsilon_{p}$ are estimated as

$$
\epsilon_{s}=\frac{\left|K_{s}^{\mathrm{BEM}}-K_{s}\right|}{K_{s}}, \quad \epsilon_{p}=\frac{\left|K_{p}^{\mathrm{BEM}}-K_{p}\right|}{K_{p}} .
$$

In Fig. 5, the variations of these two relative errors are reported as a function of the mesh density $\omega$, defined as the number of boundary elements per unit length of tile side $l_{0}$.

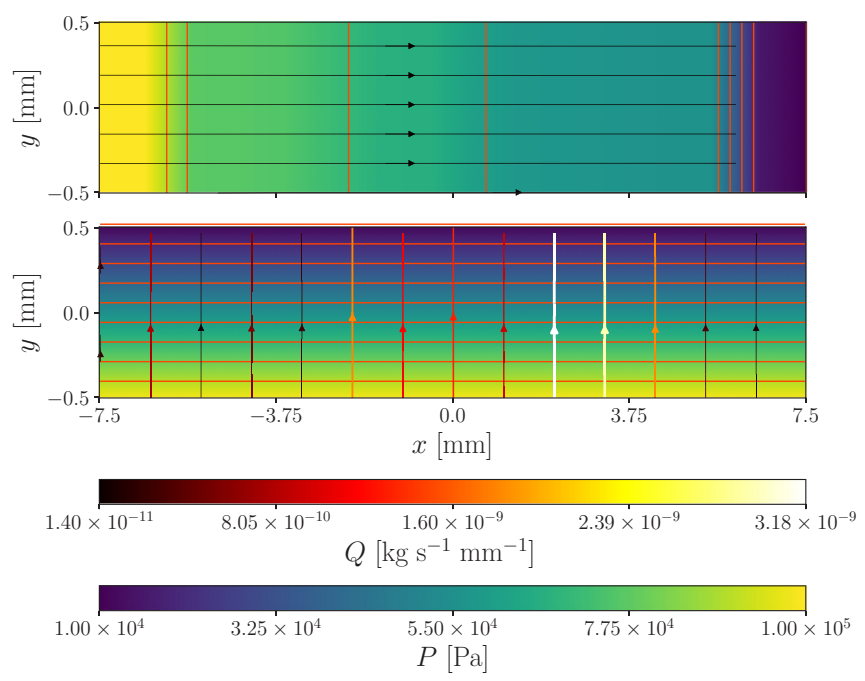

FIG. 6. Internal fields computed with the two-scale method in serial (top) and parallel (bottom) configurations. The blue to yellow color scale is used to display the pressure field. Isobars are materialized by orange lines. Arrow lines represent the streamlines of mass flow rate per unit width which color scale ranges from dark red to white.

Both errors decrease as the mesh density increases, the error in the parallel case remaining always smaller than in the serial case. To reach a relative error of $1 \%$ for this surface, around 8 elements per tile side are required in the parallel configuration whereas 16 elements are necessary in the serial case. As depicted by the dashed line, the constant boundary element scheme exhibits a power law convergence rate of order approximately equal to 1.7. A refined grid was chosen to discretize the aperture field $h(x, y)$ on each tile so as to obtain a local transmissivity with an accuracy to within machine precision while solving the closure problem in Eqs. (8) (see [15], showing an extremely fast converging computation for cosinelike surfaces). As a consequence, the convergence rate reported in Fig. 5 can be entirely attributed to the BEM scheme.

To complete this first comparison, the computed internal fields of the pressure and mass flow rate per unit length are represented in Fig. 6 for the two flow configurations. As expected, the one-dimensional flow character is clearly highlighted in this figure for both cases. The overall results validate the calculation of the transmissivity tensor on each tile [solving problem (8)] together with the BEM scheme.

\section{B. Rough surfaces}

To better assess the efficiency of the present two-scale method on general heterogeneous and anisotropic media, its predictions are further investigated on two illustrative examples and compared to reference results. These reference results are obtained by direct numerical simulation (DNS) of the Reynolds problem in Eqs. (1) at the roughness scale $l_{\beta}$ over the entire system of scale $L_{f}$, using the same set of megascale Dirichlet boundary conditions as in the two-scale method. To do so, the system of Eqs. (1) is discretized with a second-order accurate finite volume scheme and solved with the conjugate 


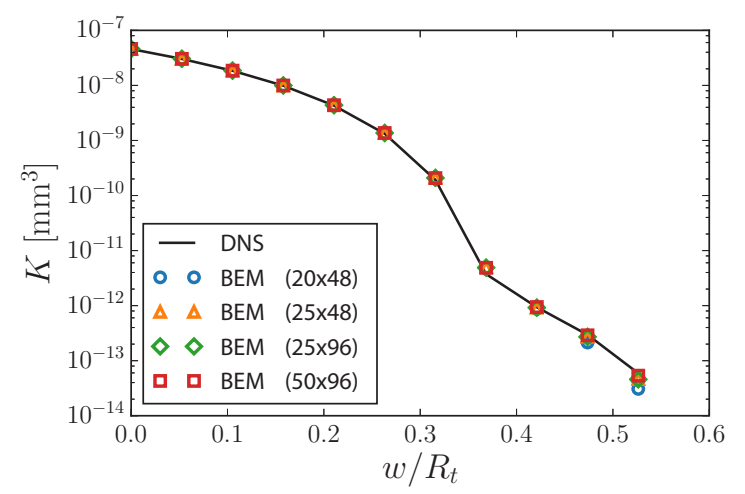

(a)

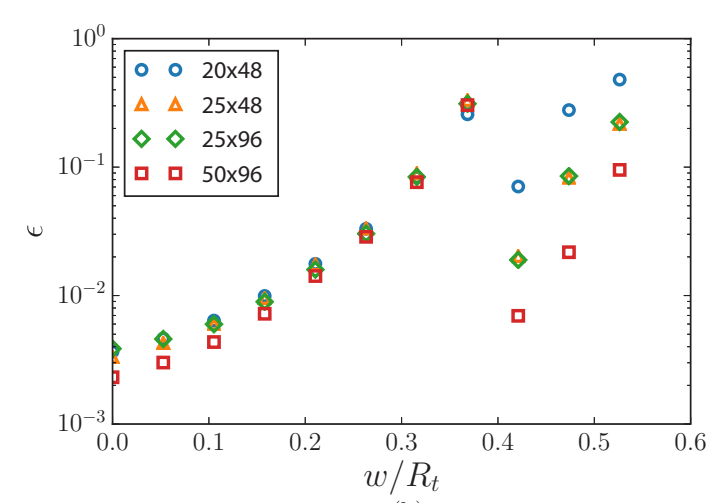

(b)

FIG. 7. Transmissivity $K$ of the spiral-groove fracture in the $x$ direction as a function of the dimensionless squeezing displacement $w / R_{t}$ obtained from DNS and the two-scale method (BEM) with four tessellations (a). Relative error on $K$ obtained from DNS and the BEM for the four tiling configurations versus $w / R_{t}$ (b).

gradient algorithm. The detailed procedure for such a solution is quite standard and will not be thoroughly described here. Further information for this numerical scheme can be found for example in [35].

\section{Spiral-groove surface}

In this first example, the case of a spiral-groove surface is considered. Such surfaces are usually obtained as a result of a face turning process, leading to a texture with a characteristic spiral groove. They find applications in the domain of static sealing with metal gaskets for instance [2,3,6,7,47].

As a model, an artificial spiral-groove surface $z(x, y)$ is synthesized. It is composed of simple sine waves on which random noise is superimposed. Generation of this surface is performed on a grid of $500 \times 4800$ points using the procedure and the values of the different parameters reported in the AppendixA. The resulting total roughness $R_{t}$ of this surface (i.e., the peak-to-peak height) is $R_{t}=17.5 \mu \mathrm{m}$. To obtain the aperture field of the fracture $h$, the spiral-groove surface is displaced downward by an amount $w$ against a nominally flat plane located at $z=0$. Instead of applying a complex mechanical deformation to the two contacting surfaces, the discussion of which is out of the scope of this work, a pure erosion simplified mechanism is used. To this end, the rough spiral-groove surface is considered as rigid while the nominally flat surface is deformed as a result of the intersection

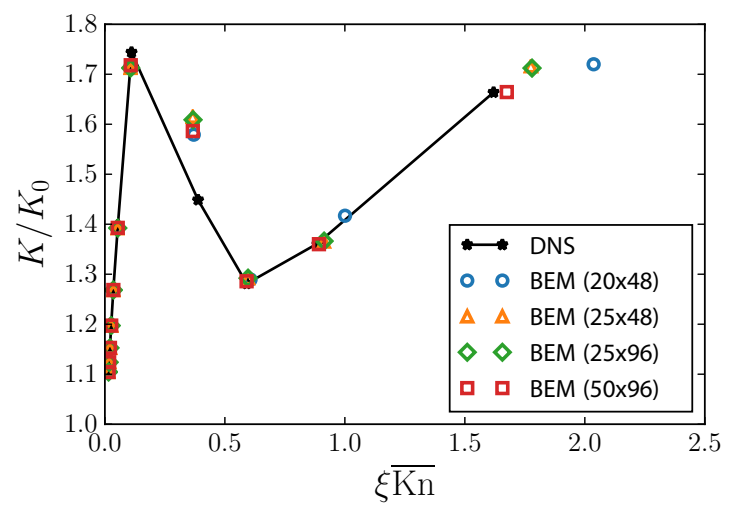

FIG. 8. Dimensionless transmissivity of the spiral-groove fracture as a function of the average Knudsen number defined in Eq. (38).

with the rough surface, yielding $h=\max (z-w, 0)$. Thus, the aperture field is created by contacting two surfaces with an external mechanical load. Since no fluid-structure interaction is considered, the aperture is then considered as fixed during fluid flow.

Once the aperture field is determined, the microscale flow can be computed by DNS on the one hand and the two-scale method (BEM) can be employed after a tessellation is chosen on the other hand. Four different tiling configurations are used for the surface under consideration, namely, $20 \times 48$, $25 \times 48,25 \times 96$, and $50 \times 96$ tiles in the $x$ and $y$ directions, respectively. Moreover, a sufficiently large number of boundary elements per tile is selected to reach convergence, which, for the four tessellations, is found to be respectively 160, 192, 112, and 112. The Dirichlet boundary conditions are such that $P_{i}=2 \times 10^{5} \mathrm{~Pa}$ and $P_{e}=10^{5} \mathrm{~Pa}$ at $x=x_{0}$ and $x_{0}+L_{x}$, respectively $\left(x_{0}=1 \mathrm{~mm}\right.$ here), while periodicity is assumed in the $y$ direction. Moreover, flow of helium $(M=$ $\left.4.0026 \times 10^{-3} \mathrm{~kg} \mathrm{~mol}^{-1}, \mu=1.8695 \times 10^{-5} \mathrm{~Pa} \mathrm{~s}\right)$ at room temperature $(T=293.15 \mathrm{~K})$ is considered with $\xi=1$ [see Eqs. (1b) and (9)] and the ideal gas law is assumed. Under these circumstances, an average Knudsen number quantifying the rarefaction effects within the entire fracture can be defined as [48]

$$
\overline{\mathrm{Kn}}=\frac{\mu}{\bar{P} h_{0}} \sqrt{\frac{\pi R T}{2 M}},
$$

where $R=8.314 \mathrm{~J} \mathrm{~K}^{-1} \mathrm{~mol}^{-1}$ is the ideal gas constant, $\bar{P}=$ $\left(P_{i}+P_{e}\right) / 2$ the mean pressure, and $h_{0}$ is a characteristic aperture of the fracture, defined as the distance between two parallel plates that would exhibit the same intrinsic transmissivity $K_{0}$ (i.e., without slip) given by

$$
h_{0}=\sqrt[3]{12 K_{0}} .
$$

The computation of $K$ is performed for $0 \leqslant w / R_{t} \leqslant 0.526$, which corresponds to $5.98 \times 10^{-2} \mu \mathrm{m} \leqslant h_{0} \leqslant 7.93 \mu \mathrm{m}$ and, hence, $1.54 \times 10^{-2} \leqslant \overline{\mathrm{Kn}} \leqslant 2.04$. The upper value obviously falls beyond the upper limit for which slip flow remains a reasonable approximation. However, this will be disregarded in the present analysis which is dedicated to the qualification of the two-scale method from a numerical point of view. It should be noted that, for a fixed value of $w / R_{t}, \overline{\mathrm{Kn}}$ in the two-scale method can be different from that obtained with 


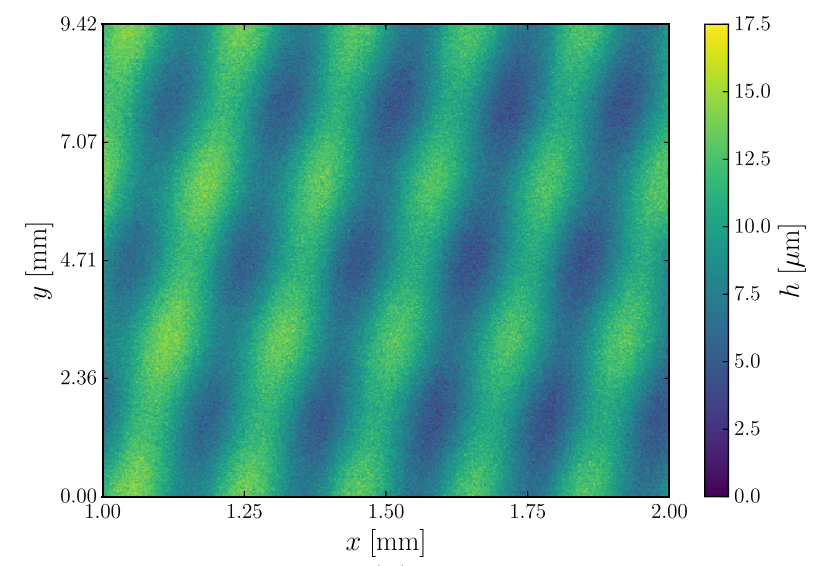

(a)

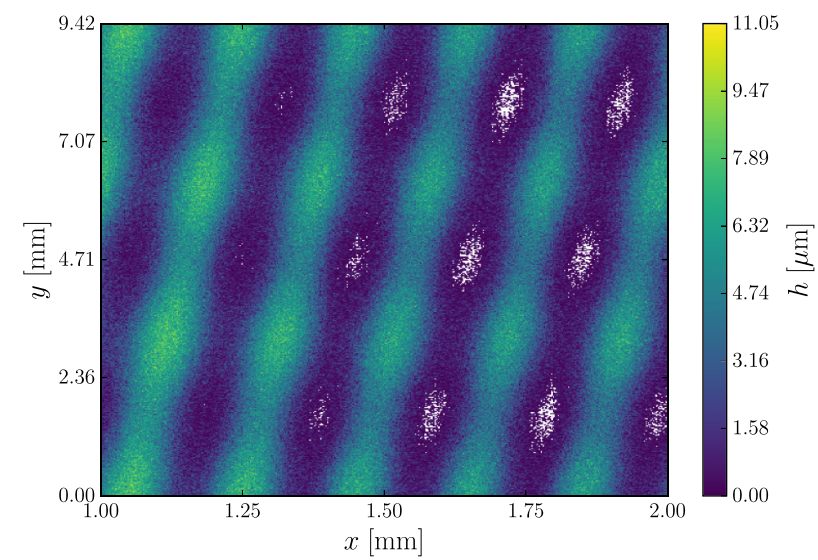

(c)

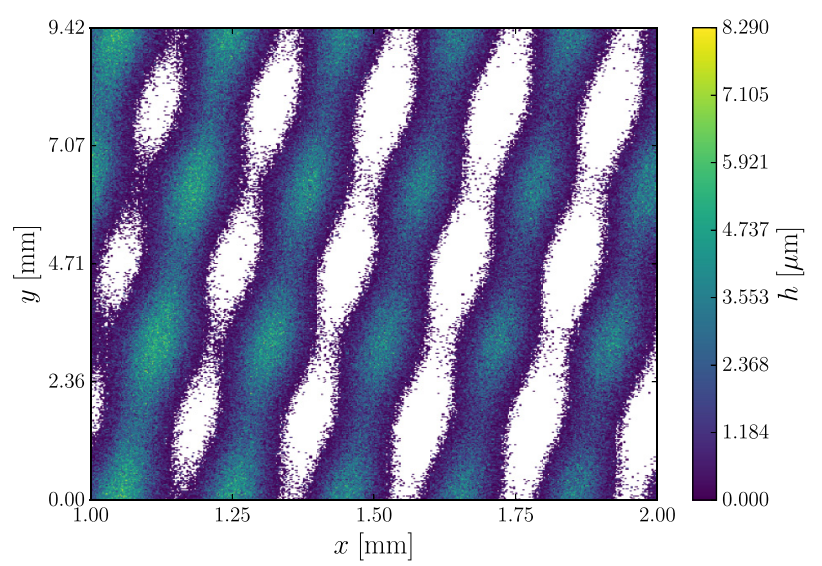

(e)

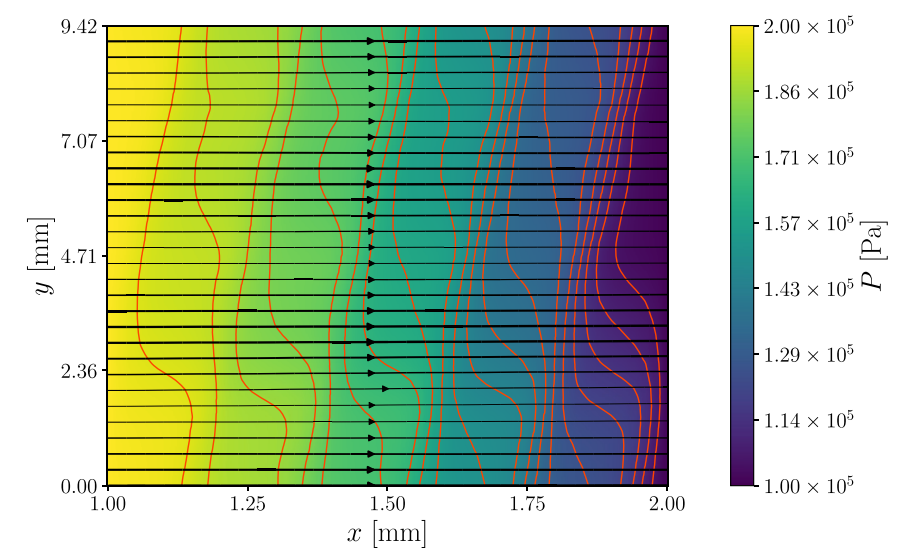

(b)

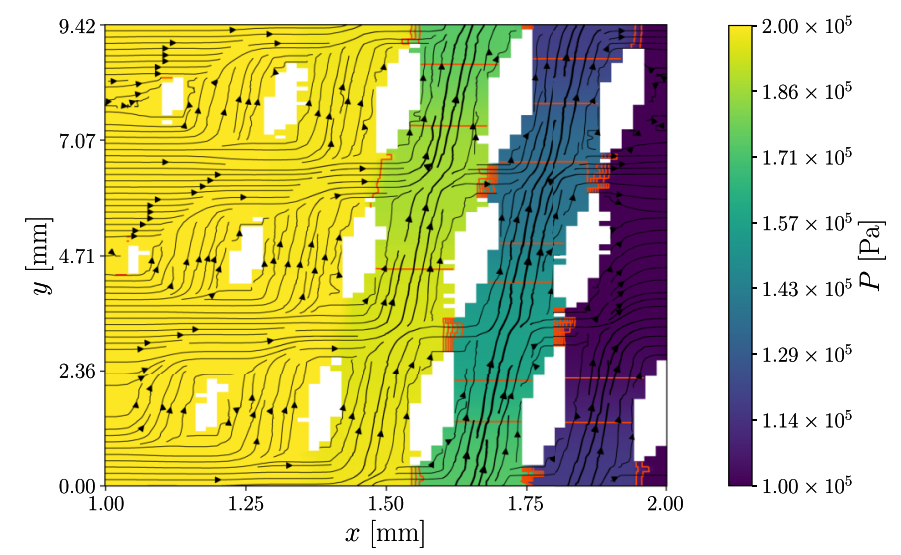

(d)

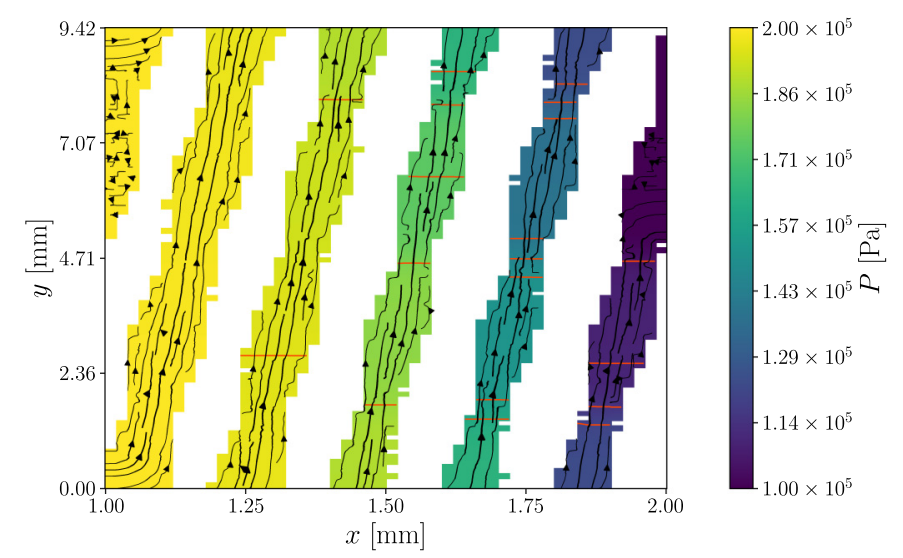

(f)

FIG. 9. Left: Aperture field of the spiral-groove surface at different squeezing displacement $w / R_{t}=0.00(\overline{\mathrm{Kn}} \approx 0.015)(\mathrm{a}) ; w / R_{t}=0.37$ $(\overline{\mathrm{Kn}} \approx 0.37)(\mathrm{c})$; and $w / R_{t}=0.53(\overline{\mathrm{Kn}} \approx 1.68)(\mathrm{e})$. White zones denote contact spots of zero aperture. Right: Color map of the computed pressure fields with superimposed isobar lines (in orange) and mass flux streamlines (arrow lines) computed with the two-scale method for the corresponding aperture fields on the left. (b) $w / R_{t}=0.00(\overline{\mathrm{Kn}} \approx 0.015)$; (d) $w / R_{t}=0.37(\overline{\mathrm{Kn}} \approx 0.37)$; (f) $w / R_{t}=0.53(\overline{\mathrm{Kn}} \approx 1.68)$. $50 \times 96$ tessellation. White zones denote nonpercolating tiles. The aspect ratio is distorted for clarity of the representation.

DNS and can vary from one tessellation to another as a result of a variation in the estimation of $K_{0}$ induced by the number of tiles.

The dependence of the transmissivity $K$ of the spiralgroove fracture in the $x$ direction, obtained from DNS and the BEM with the four tessellations, are represented versus the dimensionless displacement in Fig. 7(a) showing that $K$ decreases by roughly six orders of magnitude over the whole range of $w / R_{t}$. The decrease changes in slope at $w / R_{t} \approx 0.37$, corresponding to the "radial percolation threshold," at which the flow is forced through the groove and no direct flow in the $x$ direction is possible anymore. For larger values of $w / R_{t}$, the transmissivity decreases steadily as the flow takes place in the spiral regime. 
The relative error between the transmissivity obtained with DNS and the two-scale method is presented in Fig. 7(b). It is mostly less than $10 \%$ whatever the tessellation. The error increases when approaching the radial-to-spiral transition (when $w / R_{t} \leqslant 0.37$ ) due to the presence of radial critical constrictions. In the spiral regime (for $w / R_{t} \geqslant 0.37$ ), the error is suddenly reduced as the flow is controlled by the spiral groove. However, the error increases for larger values of the displacement, while nearing the global percolation threshold. In this last regime, increasing the number of tiles in the $x$ direction helps reducing the error, as the flow in the spiral is better described. The number of tiles in the $y$ direction does not seem to have a strong influence, at least beyond a large enough value (see results obtained with the $25 \times 48$ and $25 \times 96$ tessellations).

The dependence of the dimensionless transmissivity $K / K_{0}$, upon $\overline{\mathrm{Kn}}$ is shown in Fig. 8. A satisfactory agreement between DNS and the BEM results can be observed, the largest discrepancy occurring when the error on the computed transmissivity is noticeable, close to the radial-to-spiral transition and to the global percolation threshold as already observed in Fig. 7(b). It should be noticed that, for a fixed mean pressure, the variation of the average Knudsen number is only due to the change of the aperture field with the squeezing displacement [see Eqs. (38) and (39)]. As a consequence, the sudden change of slope sign at $\xi \overline{\mathrm{Kn}} \approx 0.2$ is a signature of the radial-to-spiral transition regime.

As an overall illustration, the different flow patterns are depicted in Fig. 9 where the fields of $h$ for three different dimensionless values of the squeezing displacement $w / R_{t}$ are represented [see Figs. 9(a), 9(c), and 9(e)] together with the corresponding pressure and flux fields [Figs. 9(b), 9(d), and 9(f), respectively] for the $50 \times 96$ tessellation. In Figs. 9(a) and 9(b), the flow is essentially radial, in the $x$ direction, as indicated by the streamlines (black arrow lines) in Fig. 9(b). For $w / R_{t} \approx 0.37$ [Figs 9(c) and 9(d)] the effective contact zones are close to form continuous clusters blocking direct flow in the $x$ direction. At this stage, the fluid is forced to partially follow the spiral groove. Further increasing the squeezing displacement leads to a pure spiral flow over the entire fracture [Figs. 9(e) and 9(f)] which is characterized by a much smaller transmissivity than in the direct flow scenario, due to the drastic increase of viscous shear induced by the increase of the solid-fluid contact area of the effective flow paths.

\section{Fractal surface}

In this second example, the case of a self-affine fractal surface is considered with a treatment similar to the spiral-groove surface of Sec. III B 1. Such rough surfaces exhibit a scale invariance under a dilatation transformation (characterized by a roughness, or Hurst exponent), a feature often found in rock cracks and faults $[49,50]$.

To begin with, a statistically isotropic self-affine surface $z(x, y)$ of roughness exponent 0.6 is generated on a grid of $2049 \times 2049$ points, using the approach and the algorithm described in [50]. The total roughness of this surface is $R_{t}=$ $17.2 \mu \mathrm{m}$. As in Sec. III B 1, a vertical rigid body displacement

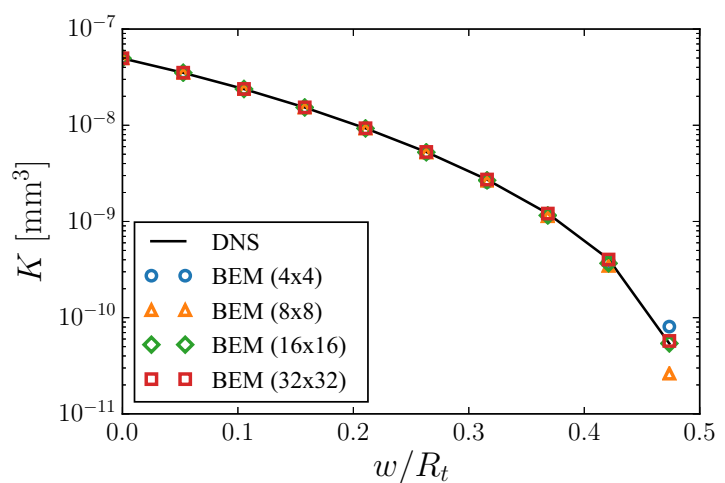

(a)

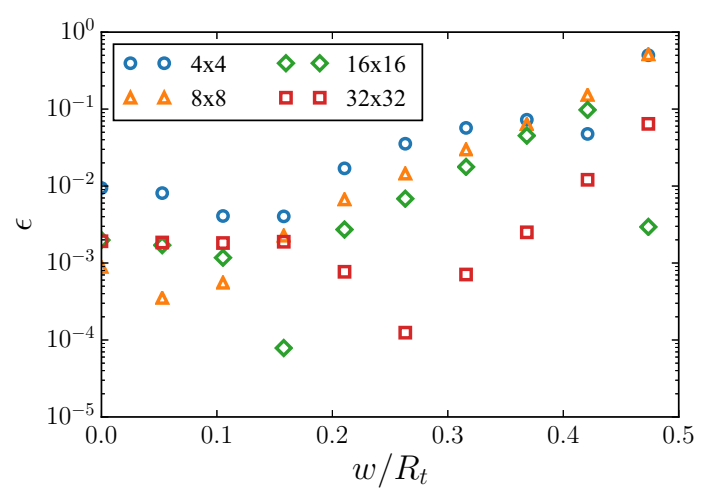

(b)

FIG. 10. Transmissivity of the fractal surface as a function of the dimensionless squeezing displacement obtained from DNS and the BEM with four tessellations (7a). Relative error on $K$ obtained from DNS and the BEM in the four tiling configurations versus $w / R_{t}(7 \mathrm{~b})$.

$w$ is then applied and the same erosion process is used to obtain the aperture field $h(x, y)=\max [z(x, y)-w, 0]$.

The aperture field being known, the flow can be computed using DNS and the two-scale method. For this fracture, four different regular tessellations are considered, namely, $4 \times 4$, $8 \times 8,16 \times 16$, and $32 \times 32$ tiles in the $x$ and $y$ directions, respectively. As for the spiral groove the number of boundary elements is selected to reach mesh convergence for a given tessellation; 64 elements per tile were found to be adequate in each case. Again, flow of an ideal gas (helium) in the same conditions as in Sec. III B 1 is considered using the same boundary conditions $\left[P_{i}=2 \times 10^{5} \mathrm{~Pa}\right.$ and $P_{e}=10^{5} \mathrm{~Pa}$ at $x=x_{0}$ and $x_{0}+L_{x}$, respectively $\left(x_{0}=0 \mathrm{~mm}\right.$ here $)$, and periodicity in the $y$ direction]. The transmissivity is computed for $0 \leqslant w / R_{t} \leqslant 0.47$. Using the definitions of $K_{0}$ and $h_{0}$ given in Eqs. (38) and (39), the corresponding ranges of the characteristic aperture and average Knudsen number are, respectively, $0.69 \mu \mathrm{m} \leqslant h_{0} \leqslant 8.20 \mu \mathrm{m}$ and $1.49 \times 10^{-2} \leqslant$ $\overline{\mathrm{Kn}} \leqslant 2.34 \times 10^{-1}$. As for the spiral-groove fracture, $\overline{\mathrm{Kn}}$ in the BEM differs from one tesselation to another (and from the value obtained with DNS) due to the difference in the estimation of the intrinsic transmissivity $K_{0}$.

In Fig. 10(a) the transmissivity of the fracture in the $x$ direction is represented as a function of the dimensionless squeezing displacement. The dependence of $K$ on $w / R_{t}$ contrasts with that obtained on the spiral-groove surface. Indeed, for the fractal fracture, $K$ varies over only three orders of 


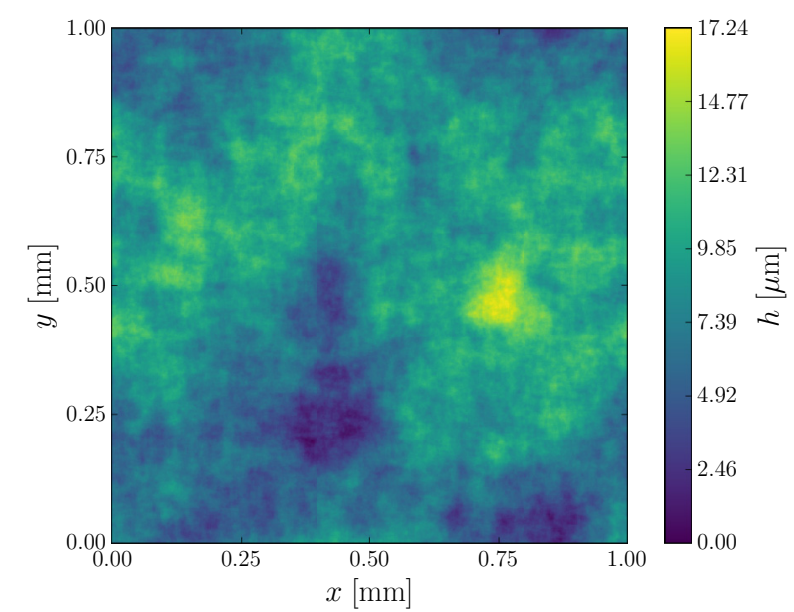

(a)

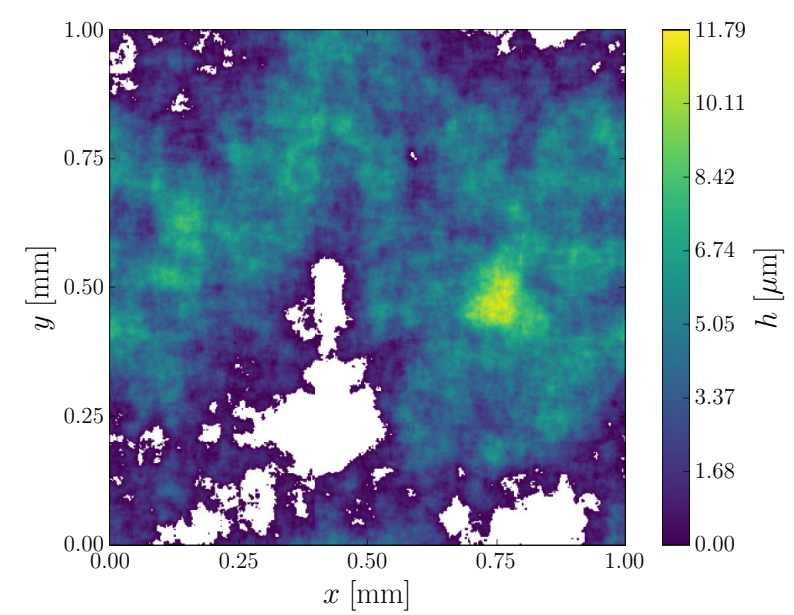

(c)

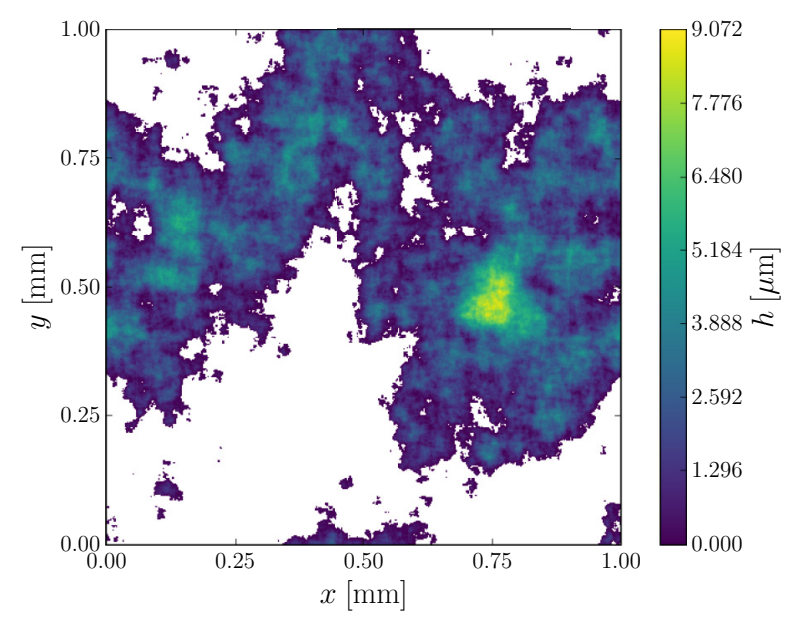

(e)
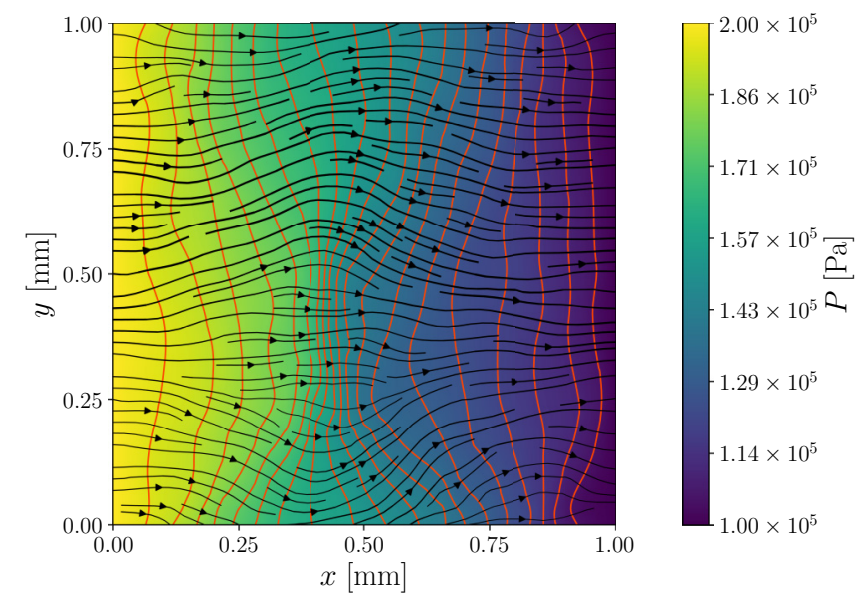

(b)

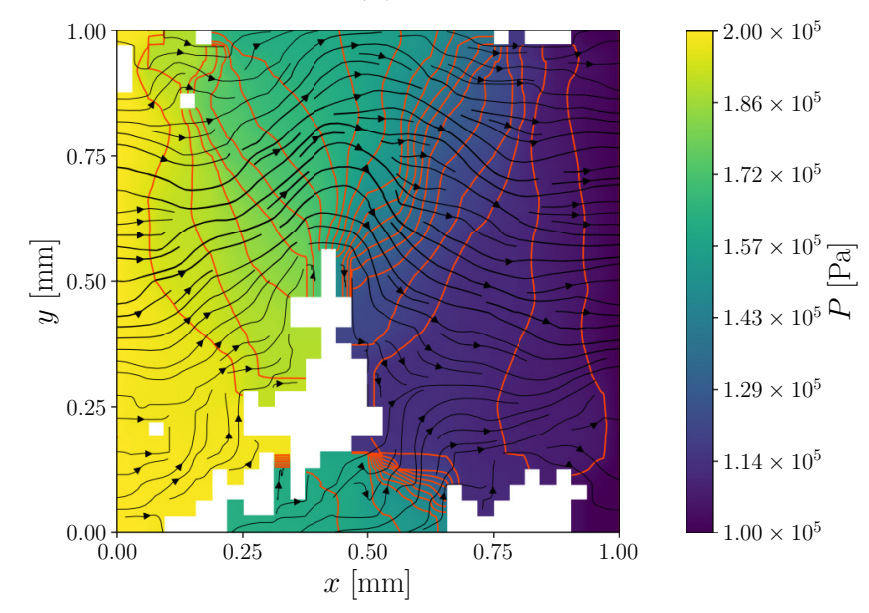

(d)

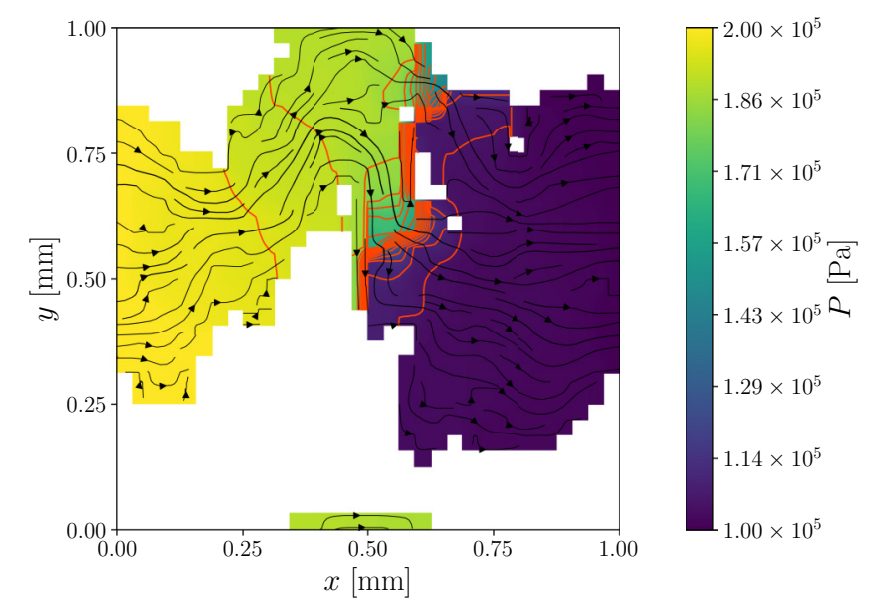

(f)

FIG. 11. Left: Aperture field of the fractal surface at different squeezing displacement $w / R_{t}=0.00(\overline{\mathrm{Kn}} \approx 0.015)(\mathrm{a}) ; w / R_{t}=0.32$ $(\overline{\mathrm{Kn}} \approx 0.041)(\mathrm{c}) ;$ and $w / R_{t}=0.47(\overline{\mathrm{Kn}} \approx 0.17)(\mathrm{e})$. White zones denote contact spots of zero aperture. Right: Color map of the computed pressure fields with superimposed isobar lines (in orange) and mass flux streamlines (arrow lines) computed with the two-scale method for the corresponding aperture fields on the left. (b) $w / R_{t}=0.00(\overline{\mathrm{Kn}} \approx 0.015)$; (d) $w / R_{t}=0.32(\overline{\mathrm{Kn}} \approx 0.041)(\mathrm{f}) w / R_{t}=0.47(\overline{\mathrm{Kn}} \approx 0.17)$. $32 \times 32$ tessellation. White zones denote nonpercolating tiles. 


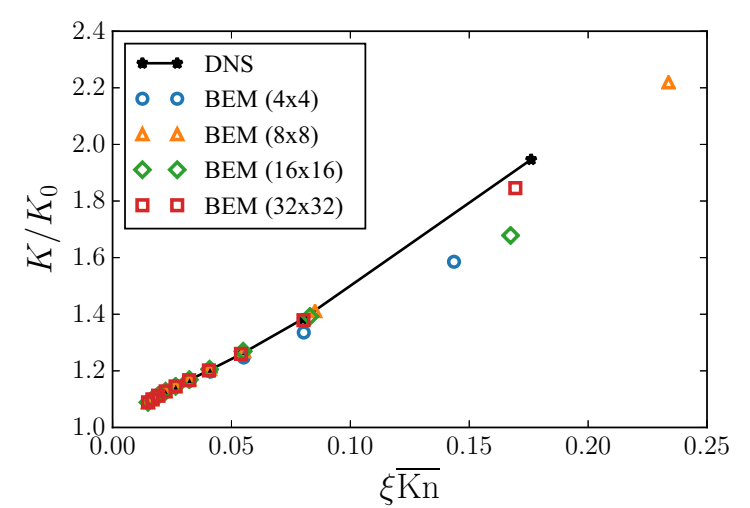

FIG. 12. Dimensionless transmissivity of the fractal surface as a function of the average Knudsen number.

magnitude (instead of six for the spiral-groove fracture) over the same range of squeezing displacement. Correspondingly, the relative error on the values of $K$ obtained from DNS and the two-scale method is reported in Fig. 10(b).

Clearly, the agreement of the two-scale method with the DNS is very satisfactory. For $w / R_{t}$ smaller than 0.2 , all paving configurations give a relative error with respect to the DNS results on $K$ less than $1 \%$. For larger values of the displacement, the error tends to increase. This is due to the increasing occurrence of critical constrictions which dominate the overall transport of the fracture. This behavior is highlighted in Fig. 11 displaying the aperture field for different values of the dimensionless squeezing displacement $w / R_{t}$ [Figs. 11(a), $11(\mathrm{c})$, and 11(e)] showing the expansion of contact zones and loss of connectivity with increasing displacements. The corresponding internal pressure fields computed by the two-scale method are presented as color maps on the right of Fig. 11 [see Figs. 11(b), 11(d), and 11(f)] using the $32 \times 32$ tessellation, together with the superimposed isobars (orange lines) and mass flux streamlines (black arrow lines). In particular, it can be observed in Fig. 11(f) that the overall pressure drop is almost concentrated at two locations in this situation where the aperture is close to the percolation threshold.

Due to the absence of a marked transition in the flow pattern, the dependence of the dimensionless transmissivity of the fractal fracture $K / K_{0}$ upon $\overline{\mathrm{Kn}}$, represented in Fig. 12, is also very different from that obtained for the spiral-groove fracture as it steadily increases with the average Knudsen number. It must also be noted that the difference between the Knudsen number estimated in the DNS approach and with the BEM remains very small, specifically in the lower range of $\overline{\mathrm{Kn}}$ (for the smallest values of $w / R_{t}$ ). In the upper range of $\overline{\mathrm{Kn}}$, this difference decreases while increasing the number of tiles confirming the consistency of the BEM. These results show the robustness of the present method to determine the transmissivity of a fracture in the slip flow regime. In particular, the use of a piecewise constant density and representative mean free path on each tile seems to have a weak influence on the estimation of the fracture transmissivity, provided the flow is not dominated by critical constrictions. In addition, it must be noticed that the method reveals to be robust in the present case of a fractal fracture for which the constraint of the lengthscales separation expressed in (4), required, in principle, to
TABLE II. Average computational time (in minutes) over the whole range of squeezing displacements (and the different tessellations reported in the last column for the BEM) of the two methods (DNS and two-scale) for the two surfaces investigated in Secs. III B 1 and III B 2. Time values in parentheses indicate the associated mean absolute deviations.

\begin{tabular}{lccc}
\hline \hline \multicolumn{3}{c}{ Time } \\
\hline Fracture & DNS & Two-scale & Tiles \\
\hline Fractal & & $49(8)$ & $4 \times 4$ \\
& \multirow{2}{*}{$121(14)$} & $24(4)$ & $8 \times 8$ \\
& & $7(1)$ & $16 \times 16$ \\
& & $6(1)$ & $32 \times 32$ \\
Spiral groove & & $5(1)$ & $20 \times 48$ \\
& $67(35)$ & $7(1)$ & $25 \times 48$ \\
& & $6(2)$ & $25 \times 96$ \\
& & $22(8)$ & $50 \times 96$ \\
\hline \hline
\end{tabular}

obtain the macroscopic equations (6), is not satisfied (i.e., a local RES may not exist for such fracture exhibiting a continuum of scale). These results might be explained by the fact that the transport behavior of a fracture is governed by the largest scales of its aperture and therefore that an approach with a tessellation, which acts a as low-pass filter, is sufficient, up to a certain point when nearing the percolation threshold. Indeed, for a fractal fracture, it was shown in [51] that the transmissivity quickly converges to its reference value while increasing the number of components in the Fourier spectrum when filtering the aperture. Moreover, it was suggested that the convergence speed increases with the roughness exponent.

\section{Comparative performance of the DNS and two-scale approaches}

In this section, a quick comparison of the performance of the DNS and the two-scale method developed in this work is shortly presented in terms of execution times of the corresponding algorithms. As an illustration, computational times are provided in Table II where the average values over the whole range of squeezing displacement (and tessellations for the BEM) are provided for both the DNS and BEM, for the spiral-groove and fractal fractures; the mean absolute deviations are indicated in parentheses. These times correspond to runs performed on the same desktop computer including four processors.

Although the code used for DNS is a pure sequential program while the MUMPS linear system solver used in the BEM is a partially built-in parallel tool, comparison between the two codes may be performed. Data in Table II clearly indicate that the two-scale method allows a speedup in the determination of the transmissivity up to a factor of 20 . Moreover, the execution time decreases while refining the tessellation (this is particularly obvious for the fractal fracture), at least up to a certain refinement. This can be explained by the fact that, increasing the number of tiles requires less time to compute the transmissivity on each of them since the number of corresponding degrees of freedom is reduced. However, this holds up to a certain refinement as can be observed for 
the finest tessellation on the spiral-groove fracture for which time increases significantly compared to coarser ones. This behavior results from the complexity of the linear system at the boundary element level (step 4 of the two-scale method) which requires more time to build the matrix and for its solution, the former operation being the most time consuming operation. From this observation, an optimal tessellation could be envisaged. In addition, an optimization of both codes could be considered.

For DNS, the linear system solution part of the code, which is performed here using an iterative conjugate gradient algorithm and represents the major fraction of the overall computational time, may be still improved. Nevertheless, it must be emphasized that in the two-scale method, the computation of the transmissivity tensors on the assembly of tiles can be fully distributed leading to a highly parallel code in its optimized version so that the speedup reported here, making the two-scale method a very effective one that outperforms the DNS, can certainly be still improved significantly.

\section{CONCLUSIONS}

In this work, a two-scale method is developed to determine the transmissivity of a fracture in one-phase slip flow conditions, a general framework which includes the particular case of no-slip flow. The model relies, a priori, on a separation of length-scales hypothesis between the smallest scale of defects (i.e., the microscale which can be typically identified as the scale of roughness) and the macroscale which characterizes defects at the immediate larger scale (as for instance the waviness). On this basis, an upscaled macroscopic Reynolds model, derived from the underlying Reynolds model at the microscale by making use of the volume averaging formalism, is employed on a tessellation of the entire fracture. Each tile is characterized by its transmissivity tensor (symmetric but fully populated) which depends on the underlying microstructure and the fluid density, taken as uniform and equal to its mean value on the tile under concern. The density value reflects the level of rarefaction on this tile through the Knudsen number.

The flow within the entire fracture subdivided in a set of tiles characterized by a piecewise heterogeneous and fully anisotropic tensor field is solved at the megascale with a boundary element method, which allows to compute the global transmissivity. This two-scale method was employed on test rough fractures, namely, a spiral-groove and a fractal fracture while varying the mean aperture, yielding a wide range of the average Knudsen number characteristic of the flow in the whole fracture. Results were compared to those obtained from a direct numerical simulation (DNS) to solve the microscale flow problem over the entire fracture. A very satisfactory agreement is obtained between the two approaches, provided the overall flow is not dominated by critical constrictions. In this case, increasing the number of tiles may help improve the solution, to the cost, however, of an increasing complexity of the method. The tests cases validate the two-scale method and prove its robustness to predict the transmissivity of the fracture. In particular, the piecewise uniform density (and mean free path) over each tile reveals to be a relevant hypothesis. Moreover, the method remains valid in the case of a fractal structure for which the separation of length scale is not fulfilled.

On the whole, the two-scale method outperforms the DNS approach allowing a very significant computational speedup which could certainly be further improved on the basis of an optimization process which could take benefit of the parallel opportunity available with this two-scale method.

\section{ACKNOWLEDGMENTS}

Financial support from CEA and CEA-Technetics Group France joint sealing laboratory for this research is gratefully acknowledged.

\section{APPENDIX}

In this Appendix, the method to synthesize the spiralgroove surface used in Sec. III B 1 is presented. The approach is quite similar to the one used in [6]. The surface profile $z(x, y)$ is described as a linear combination of typical elementary features of a spiral-groove surface, namely,

$$
z(x, y)=z_{s}+z_{c w}+z_{r w}+z_{n}
$$

with $z_{s}$ the spiral-groove profile, $z_{c w}$ the "circumferential" waviness (in the $y$ direction), $z_{r w}$ the "radial" waviness (in the $x$ direction), and $z_{n}$ the asperities of the profile given by a random noise following a normal distribution. Considering a rectangular domain of sizes $L_{x}$ and $L_{y}$ in the $x$ and $y$ directions, respectively, their expressions are given by

$$
\begin{aligned}
z_{s}= & \Upsilon_{s} \sin \left(2 \pi n_{s} \frac{x-x_{0}}{L_{x}}-2 \pi \frac{y-y_{0}}{L_{y}}+\varphi_{s}\right), \\
z_{c w}= & \Upsilon_{c w} \sin \left(2 \pi n_{c w} \frac{y-y_{0}}{L_{y}}+\varphi_{c w}\right), \\
z_{r w}= & \Upsilon_{r w} \sin \left(2 \pi n_{r w} \frac{x-x_{0}}{L_{x}}+\varphi_{r w}\right), \\
& z_{n} \sim \mathcal{N}\left(0, \sigma^{2}\right),
\end{aligned}
$$

where $\Upsilon_{i}$ and $n_{i}$ are the amplitude and wave number parameters, $\varphi_{i}$ is the phase at the origin $(i=s, c w, r w)$, while $\mathcal{N}\left(0, \sigma^{2}\right)$ is the asperities height normal distribution having a 0 mean and a root mean square $\sigma$. Values of the parameters employed to generate the surface considered in Sec. III B 1 with $L_{x}=1 \mathrm{~mm}, L_{y}=3 \pi \mathrm{mm}, x_{0}=1 \mathrm{~mm}$, and $y_{0}=0 \mathrm{~mm}$, along with $\sigma=1 \mu \mathrm{m}$, are reported in Table III.

TABLE III. Values of the different parameters $\Upsilon_{i}, n_{i}$, and $\varphi_{i}$ in Eqs. (A2) for $i=s, c w, r w$.

\begin{tabular}{lccc}
\hline \hline$i$ & $s$ & $c w$ & $r w$ \\
\hline$\Upsilon_{i}(\mu \mathrm{m})$ & 3 & 1 & 1 \\
$n_{i}$ & 5 & 3 & 0.5 \\
$\varphi_{i}(\mathrm{rad})$ & 0 & $\pi / 2$ & $3 \pi / 4$ \\
\hline \hline
\end{tabular}


[1] B. Berkowitz, Adv. Water Resour. 25, 861 (2002).

[2] C. Marie, D. Lasseux, H. Zahouani, and P. Sainsot, Eur. J. Mech. Environ. Eng. 48, 81 (2003).

[3] C. Marie and D. Lasseux, J. Fluids Eng. 129, 799 (2007).

[4] C. Vallet, D. Lasseux, P. Sainsot, and H. Zahouani, Tribol. Int. 42, 250 (2009).

[5] Y. Ledoux, D. Lasseux, H. Favreliere, S. Samper, and J. Grandjean, Int. J. Pressure Vessels Piping 88, 518 (2011).

[6] S. Geoffroy and M. Prat, J. Fluids Eng. 126, 48 (2004).

[7] F. Pérez-Ràfols, R. Larsson, and A. Almqvist, Tribol. Int. 94, 421 (2016).

[8] Y. Méheust and J. Schmittbuhl, Pure Appl. Geophys. 160, 1023 (2003).

[9] B. Lorenz and B. N. J. Persson, Europhys. Lett. 86, 44006 (2009).

[10] W. B. Dapp and M. H. Müser, Sci. Rep. 6, 19513 (2016).

[11] H. Christensen, in Proceedings of the Institution of Mechanical Engineers (Institution of Mechanical Engineers, London, 1970), pp. 1013-1026, Vol. 184.

[12] N. Patir and H. S. Cheng, J. Lubr. Technol. 100, 12 (1978).

[13] J. H. Tripp, J. Lubr. Technol. 105, 458 (1983).

[14] M. Prat, F. Plouraboué, and N. Letalleur, Transp. Porous Media 48, 291 (2002).

[15] T. Zaouter, D. Lasseux, and M. Prat, J. Fluid Mech. 837, 413 (2018).

[16] Y. W. Tsang and C. F. Tsang, Water Resour. Res. 23, 467 (1987).

[17] K. J. Stout, E. J. Davis, and P. J. Sullivan, Atlas of Machined Surfaces (Springer, Berlin, 1990).

[18] K. J. Stout and L. Blunt, Three Dimensional Surface Topography (Elsevier, Amsterdam, 2000).

[19] J. Bourniquel, D. Lasseux, and J. F. Rit, J. Press. Vessel Technol. 140, 051203 (2018)

[20] F. Pérez-Ràfols, R. Larsson, S. Lundström, P. Wall, and A. Almqvist, Proc. R. Soc. A 472, 20160069 (2016).

[21] G. E. Karniadakis, A. Beskok, and N. Aluru, Microflows and Nanoflows: Fundamentals and Simulation, Interdisciplinary Applied Mathematics (Springer, New York, 2005), Vol. 29.

[22] E. B. Arkilic, M. A. Schmidt, and K. S. Breuer, J. Microelectromech. Syst. 6, 167 (1997).

[23] A. Beskok and G. E. Karniadakis, Microscale Thermophys. Eng. 3, 43 (1999).

[24] L. J. Klinkenberg, Drilling and Production Practice (American Petroleum Institute, Washington, DC, 1941), pp. 200-213.

[25] D. Lasseux, F. J. Valdes Parada, J. A. Ochoa Tapia, and B. Goyeau, Phys. Fluids 26 (2014).

[26] D. Lasseux, F. J. Valdes Parada, and M. L. Porter, J. Fluid Mech. 805, 118 (2016).
[27] B. J. Hamrock, S. R. Schmid, and B. O. Jacobson, Fundamentals of Fluid Film Lubrication, Mechanical Engineering (CRC Press, Boca Raton, FL, 2004).

[28] V. Mourzenko, J.-F. Thovert, and P. Adler, J. Phys. II 5, 465 (1995).

[29] J. C. Maxwell, Philos. Trans. R. Soc. London 170, 231 (1879).

[30] E. B. Arkilic, K. S. Breuer, and M. A. Schmidt, J. Fluid Mech. 437, 29 (2001).

[31] L. B. Loeb, The Kinetic Theory of Gases, Dover Phoenix Editions (Dover, New York, 2004), p. 736.

[32] A. Z. Szeri, Fluid Film Lubrication (Cambridge University Press, Cambridge, 1998).

[33] S. Whitaker, The Method of Volume Averaging, Theory and Applications of Transport in Porous Media (Springer, Berlin, 1999), Vol. 13.

[34] W. G. Gray, Chem. Eng. Sci. 30, 229 (1975).

[35] F. Moukalled, L. Mangani, and M. Darwish, The Finite Volume Method in Computational Fluid Dynamics, Fluid Mechanics and Its Applications (Springer, Berlin, 2016), Vol. 113.

[36] P. S. Huyakorn and G. F. Pinder, Computational Methods in Subsurface Flow (Academic, New York, 1983).

[37] R. W. Zimmerman and G. S. Bodvarsson, Transp. Porous Media 23, 1 (1996).

[38] J. Hoshen and R. Kopelman, Phys. Rev. B 14, 3438 (1976).

[39] O. E. Lafe, J. A. Liggett, and P. L.-F. Liu, Water Resour. Res. 17, 1431 (1981).

[40] K. Rafiezadeh and B. Ataie-Ashtiani, Eng. Anal. Boundary Elements 36, 812 (2012).

[41] K. Rafiezadeh and B. Ataie-Ashtiani, Eng. Anal. Boundary Elements 37, 527 (2013).

[42] D. Lasseux and F. J. Valdes Parada, Phys. Fluids 29 (2017).

[43] L. J. Durlofsky, Water Resour. Res. 27, 699 (1991).

[44] G. Beer, I. Smith, and C. Duenser, The Boundary Element Method with Programming (Springer, Berlin, 2008).

[45] P. R. Amestoy, I. S. Duff, J. Koster, and J.-Y. L'Excellent, SIAM J. Matrix Anal. Appl. 23, 15 (2001).

[46] P. R. Amestoy, A. Guermouche, J.-Y. L'Excellent, and S. Pralet, Parallel Computing 32, 136 (2006).

[47] F. Robbe-Valloire and M. Prat, Wear 264, 980 (2008).

[48] C. Cercignani, The Boltzmann Equation and its Applications (Springer, Berlin, 1988).

[49] F. Plouraboué, P. Kurowski, J.-P. Hulin, S. Roux, and J. Schmittbuhl, Phys. Rev. E 51, 1675 (1995).

[50] T. Candela, F. Renard, M. Bouchon, A. Brouste, D. Marsan, J. Schmittbuhl, and C. Voisin, Pure Appl. Geophys. 166, 1817 (2009).

[51] A. Neuville, R. Toussaint, and J. Schmittbuhl, Geophys. J. Int. 186, 1064 (2011). 